\title{
Novel crosstalk between Vps26a and Nox4 signaling during neurogenesis
}

\author{
Seon-A Choi ${ }^{1,2,3} \cdot$ Young-Hyun Kim ${ }^{2,4} \cdot$ Young-Ho Park ${ }^{1,2,4} \cdot$ Hae-Jun Yang ${ }^{1,2} \cdot$ Pil-Soo Jeong ${ }^{1,2} \cdot$ Jae-Jin Cha ${ }^{1,2}$. \\ Seung-Bin Yoon ${ }^{1,2} \cdot \mathrm{Ji}_{\text {-Su Kim }}^{1,2,4} \cdot$ Bong-Seok Song ${ }^{1,2,4} \cdot$ Jong-Hee Lee ${ }^{1,2} \cdot$ Bo-Woong Sim $^{1,2} \cdot$ Jae-Won Huh $^{1,2,4}$. \\ In-Sung Song ${ }^{5}$ Sang-Rae Lee ${ }^{1,2,4} \cdot$ Min-Kyu Kim $^{3} \cdot$ Jin-Man Kim ${ }^{6} \cdot$ Yun Soo Bae ${ }^{7}$ Kazuhiko Imakawa ${ }^{8,9}$. \\ Sun-Uk Kim ${ }^{1,2,4} \cdot$ Kyu-Tae Chang ${ }^{2,4}$
}

Received: 20 March 2018 / Revised: 21 September 2018 / Accepted: 28 September 2018 / Published online: 21 November 2018

(c) The Author(s) 2018. This article is published with open access

\begin{abstract}
Despite numerous studies on the molecular switches governing the conversion of stemness to differentiation in embryonic stem cells (ESCs), little is known about the involvement of the retromer complex. Under neural differentiation conditions, Vps26a deficiency ( $V p s 26 a^{-1}$ ) or knockdown suppressed the loss of stemness and subsequent neurogenesis from ESCs or embryonic carcinoma cells, respectively, as evidenced by the long-lasting expression of stemness markers and the slow appearance of neuronal differentiation markers. Interestingly, relatively low reactive oxygen species (ROS) levels were generated during differentiation of Vps26a ${ }^{-/-}$ESCs, and treatment with an antioxidant or inhibitor of NADPH oxidase (Nox), a family of ROS-generating enzymes, led to restoration of stemness in wild-type cells to the level of $V p s 26 a^{-/-}$cells during neurogenesis. Importantly, a novel interaction between Vps26a and Nox4 linked to the activation of ERK1/2 depended highly on ROS levels during neurogenesis, which were strongly suppressed in differentiating $V p s 26 a^{-/}$ESCs. Moreover, inhibition of phosphorylated ERK1/2 (pERK1/2) resulted in decreased ROS and Nox4 levels, indicating the mutual dependency between pERK1/2 and Nox4-derived ROS during neurogenesis. These results suggest that Vps26a regulates stemness by actively cooperating with the Nox4/ROS/ERK1/2 cascade during neurogenesis. Our findings have important implications for understanding the regulation of stemness via crosstalk between the retromer molecule and redox signaling, and may contribute to the development of ESC-based therapeutic strategies for the mass production of target cells.
\end{abstract}

\section{Introduction}

Embryonic stem cells (ESCs), which are derived from the inner cell mass of blastocyst stage embryos, are capable of unlimited proliferation and can differentiate into multiple cell

Edited by R.A. Knight

These three authors contributed equally: Seon-A Choi, Young-Hyun Kim, Young-Ho Park

Electronic supplementary material The online version of this article (https://doi.org/10.1038/s41418-018-0226-0) contains supplementary material, which is available to authorized users.

Sun-Uk Kim sunuk@kribb.re.kr

Kyu-Tae Chang changkt@kribb.re.kr

Extended author information available on the last page of the article lineages [1]. Their self-renewal capabilities and pluripotency are representative features of ESCs, making them a robust and suitable model to aid our understanding of developmental biology. They are also a useful resource for the development of therapeutic strategies for incurable degenerative diseases. There have been many studies of the regulation of self-renewal and specific lineage differentiation, however, relatively little is known about the underlying mechanisms. Thus, further clarification of the molecular mechanisms governing the selfrenewal and differentiation processes of ESCs is needed to yield effective target cells for academic and industrial areas.

The mammalian retromer complex consists of two subcomplexes: a vacuolar protein sorting (Vps) trimer of $\mathrm{Vps} 26$, Vps29, and Vps35 responsible for cargo selection, and a sorting nexin (Snx) dimer of Snx1, Snx2, Snx5, and Snx6 [2], presumably responsible for tubule or vesicle formation. The retromer complex functions as a key component of the endosomal protein sorting machinery, particularly in the endosome-to-Golgi retrieval pathway [3, 4], and participates 
in many biological events, such as development and disease [5-7]. Indeed, the retromer complex has been found to be closely associated with pathogenic conditions such as Alzheimer's disease through mediation of the localization of various membrane proteins, including amyloid precursor protein [8]. Furthermore, early postimplantation development in mice required the involvement of the retromer complex, as evidenced by the embryonic lethality of Snx- or Vps26a-null embryos at days 9.5 and 11.5 , respectively [9, 10]. In particular, the role of Vps26a appeared to be associated with the development of the embryonic ectoderm, which gives rise to neural-lineage cells [11, 12]. Despite compelling evidence of the involvement of Vps26a during the early postimplantation period, the underlying molecular mechanism(s) remain largely unknown.

Reactive oxygen species (ROS) are created via activation of two representative systems, namely, the activation of membrane-associated NADPH oxidases (Noxs) and mitochondrial respiratory chains, and are also secondary messengers in numerous signaling pathways, as well as being molecules responsible for cellular damage [13]. Recently, Nox-generated ROS have been suggested as important regulators or determinants of stem cell behaviors, such as the maintenance of stemness and differentiation into a variety of cell types [14, 15]. As seen in the results from a variety of mammalian cell studies, increased ROS generation leads to the activation of redox signaling pathways, including mitogenactivated protein kinase (MAPK) cascades in differentiating ESCs [16, 17], which included extracellular signal-related protein kinase1/2 (ERK1/2), c-Jun $\mathrm{NH}_{2}$-terminnal kinase (JNK), and p38 MAPK $[18,19]$. Despite numerous studies on the link between MAPKs and ESC-mediated differentiation, no relationship has been found between redox signaling and the retromer complex in mammalian systems, including ESCs.

In this study, the null mutation retromer complex molecule Vps26a $\left(V_{p s} 26 a^{--}\right)$inhibited neurogenesis of ESCs through potentiation of ESC stemness. Interestingly, ROS generation was severely impaired in differentiating $\operatorname{Vps} 26 a^{-/-}$ESCs. It is important to note that Vpa26a-dependent stemness/neurogenesis transition was tightly associated with the synergistic cooperation between Nox-derived ROS and the ERK1/2 pathway. These findings increase our understanding of retromer complex- and redox signaling-associated developmental events, including the maintenance of ESC stemness.

\section{Results}

\section{Vps26a-deficiency inhibits the loss of stemness and subsequent neurogenesis from ESCs}

To determine whether Vps26a is involved in the regulation of stemness and differentiation, ESCs were established from wild-type (WT) and Vps26a $6 a^{-/}$blastocysts at 3.5 days post coitum by expanded culture on mitotically-inactivated mouse embryonic fibroblast feeder layers (Supplementary Fig. 1), and were committed into neural-lineage cells by cultivation in neurobasal medium (NBM). Compared with the WT, colonies with an ES-like morphology and alkaline phosphatase (AP) activity were detected more frequently in differentiating Vps $26 a^{-/}$ESCs (Fig. 1a, b). Consistent with this, the proportion of colonies with strong AP activity was greater in Vps26a $a^{-/-}$cells than in WT cells (Fig. 1b). Additionally, the number of cells continuously increased in the $V p s 26 a^{-/}$group compared with WT during neural differentiation from ESCs (Fig. 1c). Semi-quantitative polymerase chain reaction (qPCR) and western blot analyses confirmed that the expression levels of the ESC stemness markers Oct $3 / 4$ and Nanog did not decrease gradually in differentiating $V p s 26 a^{-/}$cells compared with WT cells (Fig. 1d-i). Similarly, cells with strong Oct3/4 immunoreactivity were frequently detected in differentiating $V p s 26 a^{-/}$cells, whereas MAP2-postive cells were clearly stained in WT cells after 6 days of neural differentiation (Fig. 1f-i). Furthermore, HIF2 $\alpha$, a transcription factor of Oct $3 / 4$ and Nanog [8], was upregulated in $V p s 26 a^{-1-}$ cells (Supplementary Fig. 2a and 2c). During ESC-mediated neurogenesis, no differences were found in the expression of retromer complex genes, regardless of the genotype (Supplementary Fig. 3). Vps35 knockdown influence the levels of retromer complex components including Vps 26 and Vps29 [20], transfection of siVps35 decreased the Vps26a transcript level in P19 ECCs. Although knockdown of Vps35 slightly affected the maintenance of Oct3/4 in neurogenesis it did not significantly affect MAP2 (Supplementary Fig. 4).

To determine the involvement of Vps26a in regulation of the stemness/differentiation transition, WT and $V p s 26 a^{-/-}$ ESCs were proliferated anchorage-independently, leading to the formation of an embryoid body (EB), after which ESC stemness and three germ layer marker expression levels were quantified by semi-qPCR, qPCR, and western blot analyses. Under microscopic examination, a relatively small cavity size was observed in EBs compared with WT cells (Supplementary Fig. 4a). In addition, semi-qPCR and qPCR analyses revealed that transcription levels of the ESC stemness markers Oct3/4 and Nanog were maintained at higher levels in $\mathrm{Vps} 26 \mathrm{a}^{-/-}$EBs during an expanded culture period than in WT cells, whereas transcription levels of three germ layer markers for the ectoderm (MAP2, Nestin, and Fgf5), mesoderm (Brachyury), and endoderm (Gata6 and Laminin B1) were decreased in Vps26-1 EBs (Supplementary Fig. $5 \mathrm{~b}$ and $5 \mathrm{c}$ ). Consistent with these results, western blot analysis revealed that $V p s 26 a^{-/-}$EBs displayed an increase in the ESC stemness markers Oct $3 / 4$ and Nanog and a decrease in the neural differentiation markers MAP2 
a

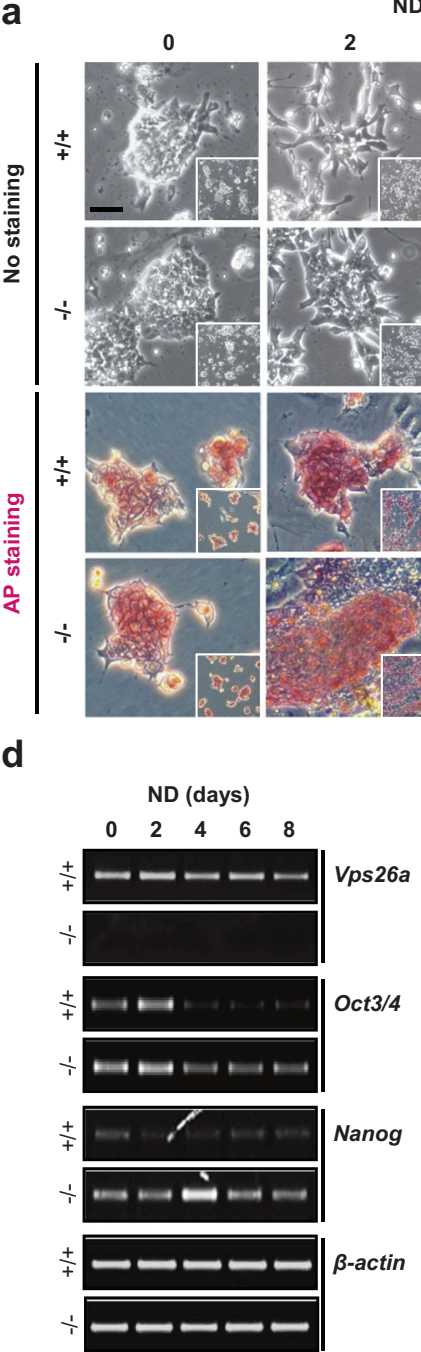

ND (days)

4
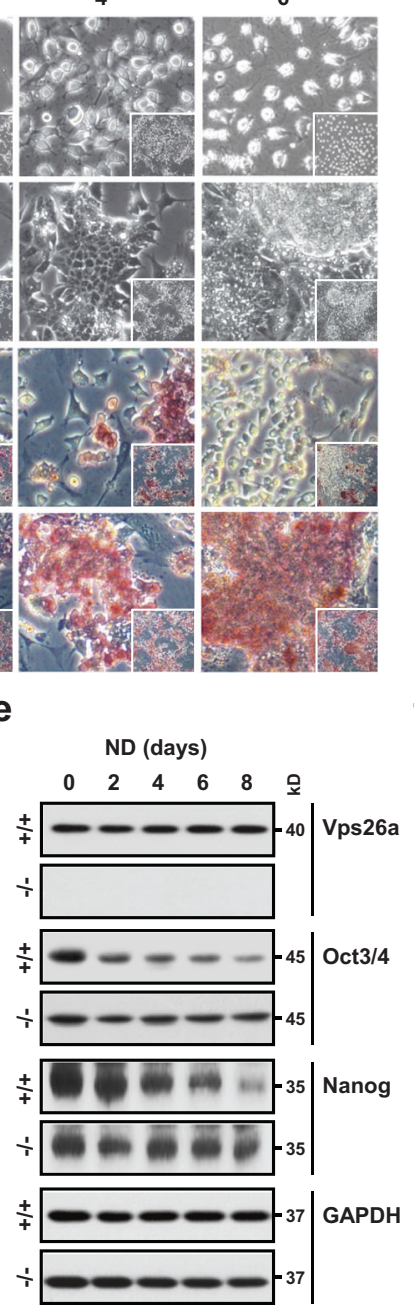

b
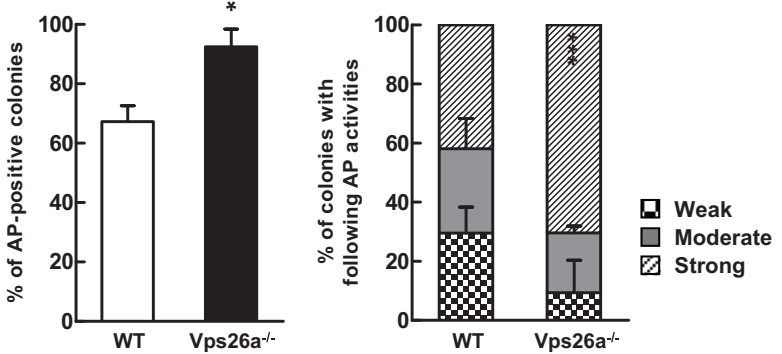

C

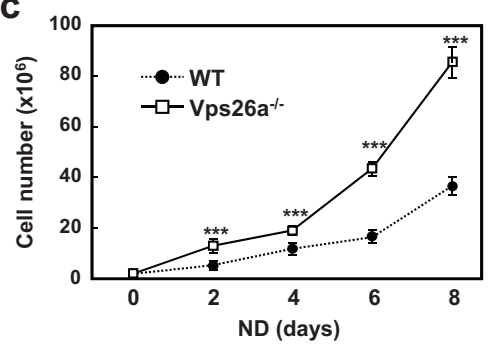

f
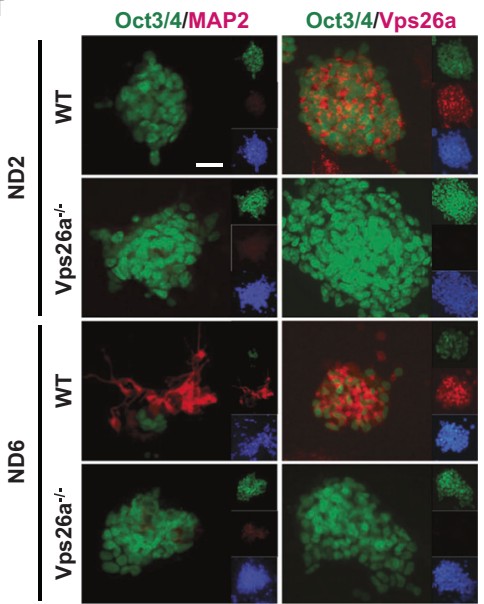

f-i

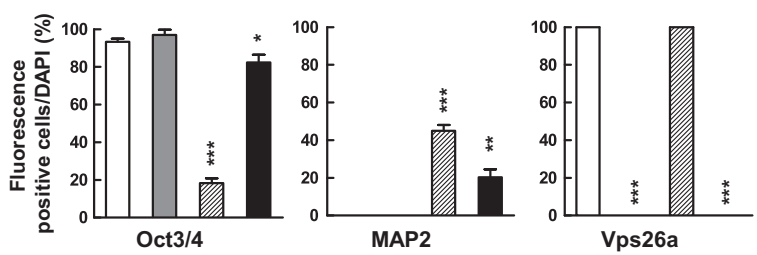

Fig. $1 \mathrm{Vps} 26 \mathrm{a}$ deficiency leads to the maintenance of stemness during ESC-mediated neurogenesis. a Effect of Vps26a deficiency on changes in alkaline phosphatase (AP) staining during neural differentiation (ND). Wild-type (WT; +/+) and Vps $26 a^{-/}(-/-)$ESCs were differentiated in neurobasal medium (NBM) for the indicated time periods and subjected to AP staining. Cell clusters with differentiated morphologies are indicated by yellow dotted lines. Scale bar, $50 \mu \mathrm{m}$. b AP activities of WT and Vps $26 a^{-1-}$ ESCs differentiated for 6 days indicated by scoring of the signal intensities of at least 60 colonies from three independent experiments. c The number of WT and $V p s 26 a^{-/-}$cells during ND for 8 days. Error bars indicate the means \pm standard deviation (SD; $n=3$ ). ${ }^{* * *} P<0.001$ compared with WT cells each day during ND. d, e Effect of Vps26a deficiency on expression

(neuron), GFAP (astrocyte), and GalC (oligodendrocyte) (Supplementary Fig. 5D). Although the size and volume of levels of ESC stemness-associated genes. $+/+$ and -/- ESCs cultured in NBM for the indicated days analyzed by semi-quantitative polymerase chain reaction (semi-qPCR) (d) and western blotting (e) analyses of Oct3/4 and Nanog. $\beta$-actin and GAPDH were used as loading controls. e-i Immunoblotting quantification of (e). Quantification of band density relative to GAPDH control ( $n=3$ for each group). Error bars are \pm SD. ${ }^{*} P<0.05 ;{ }^{* * *} P<0.001$ compared with $+/+$ cells each day during ND. f Double-label immunocytochemical analysis of Oct3/ 4 (green) and MAP2 (red) during neural differentiation for 2 or 6 days. DAPI staining data are shown as insets to the merged images. Scale bar, $50 \mu \mathrm{m}$. $\mathbf{f}-\mathbf{i}$ Quantitative analysis of the fluorescence. Percentage of fluorescence/DAPI-positive cells ( $n=3$ for each group). Error bars are \pm SD. ${ }^{*} P<0.05 ;{ }^{* *} P<0.01 ;{ }^{* * *} P<0.001$ vs. WT ND 2 days

teratomas derived from $V p s 26 a^{-/-}$ESCs were significantly larger than those of WT, WT and $V p s 26 a^{-/-}$teratomas were 
observed in all representative derivatives of the three germ layers, including the neural rosette (ectoderm), cartilage (mesoderm), and respiratory epithelium (endoderm) (Supplementary Fig. 5a-5c). Moreover, transcription levels of the stemness markers in Vps26a ${ }^{-/}$teratomas were maintained at a higher level than those in WT teratomas, indicating that $V p s 26 a^{-/-}$ESCs exhibit differentiation resistance during teratoma formation (Supplementary Fig. 6d).

\section{Vps26a knockdown delayed the loss of stemness and subsequent neurogenesis in P19 embryonic carcinoma cells}

The involvement of Vps26a in regulation of the stemness/ differentiation transition was demonstrated using neurallydifferentiating ESCs. For confirmation, another stem cell type, P19 mouse embryonic carcinoma cells (ECCs), stably transfected with short hairpin RNA (shRNA) targeting $V p s 26 a$ (shVps26a-ECCs), were neurally differentiated, and the expression levels of the stemness and differentiation markers were determined. Compared with the control shRNA-transfected ECCs (shCTL-ECCs), the Vpa26a transcription and protein levels decreased to almost zero in shVps26a-ECCs (Fig. 2a, b). In contrast, Oct3/4 and Nanog were markedly upregulated in shVps26a-ECCs compared with the control. Similar to the ESCs, cell types with morphological changes were rarely detected in neurallydifferentiating shVps26a-ECCs compared with the control (Fig. 2c). Semi-qPCR and qPCR analyses revealed that the transcription levels of Oct3/4 and Nanog decreased gradually in differentiating shVps26a-ECCs and augmented expression of the differentiation markers MAP2 and Tubb3 (also known as TuJ1) was retarded (Fig. 2d, e). Consistent with these results, long-lasting expression patterns of Oct3/ 4 and Nanog proteins were detected mostly in differentiating shVps26a-ECCs, whereas upregulation of Tubb3 was delayed compared with the control (Fig. 2f-i). Similarly, strong immunoreactivity against Tubb3 was detected in neurally differentiated shCTL-ECCs, whereas Oct3/4-positive cells were still dominant in differentiating shVps26aECCs (Fig. 2g). Furthermore, Hif $2 \alpha$, a transcription factor of Oct $3 / 4$ and Nanog, was upregulated in $V p s 26 a^{-/}$cells (Supplementary Fig. 2b). Consistent with the neurogenesis data, glial differentiation was also significantly reduced in differentiating Vps $26 \mathrm{a}^{-/}$cells compared with the control (Supplementary Fig. 7).

\section{ERK1/2 participates in Vps26a-dependent regulation of the stemness/differentiation transition during neurogenesis from ESCs}

Previous studies have shown that phosphoinositide 3-kinase (PI3K) and MAPKs are involved in ESC differentiation processes [18]. Therefore, to investigate the signaling pathways governing neurogenesis, WT and $V p s 26 a^{-1-}$ ESCs were differentiated in NBM and subjected to western blot analysis of phosphorylated ERK1/2 (pERK1/2), pp38 MAPK, pJNK, and pAKT. Of the kinases investigated, the most notable change in phosphorylation was detected in the pERK1/2 immunoblot during neural differentiation from ESCs (Fig. 3a-i). The gradual increase in pERK1/2 was markedly reduced in differentiating $V p s 26 a^{-/}$ESCs compared with WT cells (Fig. 3a-i). A similar pattern of pERK1/2 expression was also present upon comparison of shCTL- and shVps26a-ECCs (Fig. 3b-i). Consistent with these results, the overexpression of Vps26a in human embryonic kidney (HEK) 293 cells increased pERK1/2 levels in response to epidermal growth factor (Supplementary Fig. 8). To examine the suppressive effects of redoxsensitive signaling pathways on the maintenance of stemness, Vps $26 a^{-1-}$ ESCs and shVps26a-ECCs were differentiated in the presence or absence of three MAPK and AKT signaling inhibitors. Next, Oct $3 / 4$ and Nanog expression levels were examined by western blot analysis. Interestingly, PD98059 and U0126, potent ERK1/2 inhibitors, upregulated Oct3/4 and Nanog levels in normal, Vps26a-knockout, or Vps26a-knockdown cases, except for Nanog levels in WT cells (Fig. 3c, d). Consistent with these results, immunocytochemistry analysis revealed restoration of Oct3/4 immunoreactivity and a decrease in MAP2positive cells in WT cells treated with ERK1/2 inhibitors (Fig. 3e). Similarly, Oct3/4 immunoreactivity was markedly increased following treatment with ERK1/2 inhibitors in differentiating Vps26a ${ }^{-/-}$ESCs (Fig. 3e).

\section{ROS are involved in Vps26a-mediated regulation of the stemness/differentiation transition, possibly by activating Nox4 during neurogenesis from ESCs}

Evidence suggests a close association between Vps26a and several pathological conditions, including degenerative neurological diseases [12,21]. Additionally, excessive ROS are often viewed as the hallmark of a variety of degenerative diseases, with the redox status acting as a central modulator of the stemness/differentiation transition in differentiating ESCs [19]. Furthermore, numerous reports have demonstrated that ROS are involved in the activation of MAPK cascades [16, 17]. Based on these findings, ROS levels were measured in undifferentiated and differentiating ESCs stained with CM- $\mathrm{H}_{2}$ DCFDA, an indicator of ROS, by flow cytometry. ROS levels were elevated during neural differentiation from ESCs (Fig. 4a). Interestingly, Vps26a deficiency caused a decrease in ROS levels in undifferentiated and differentiating ESCs compared with WT cells (Fig. 4a), as evidenced by flow cytometric analysis using shCTL- and shVps26a-ECCs (Fig. 4b). Moreover, the reduction in ROS 
a

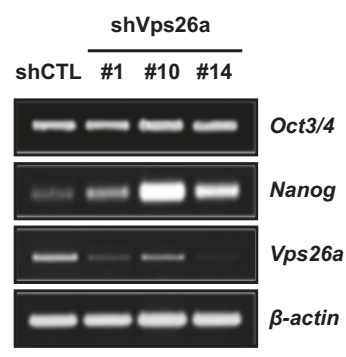

d

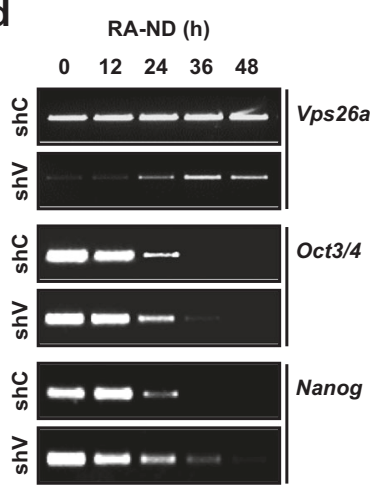

b
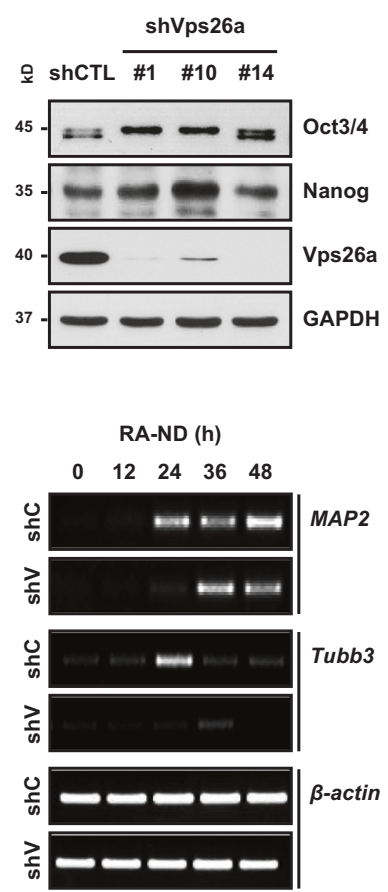

C

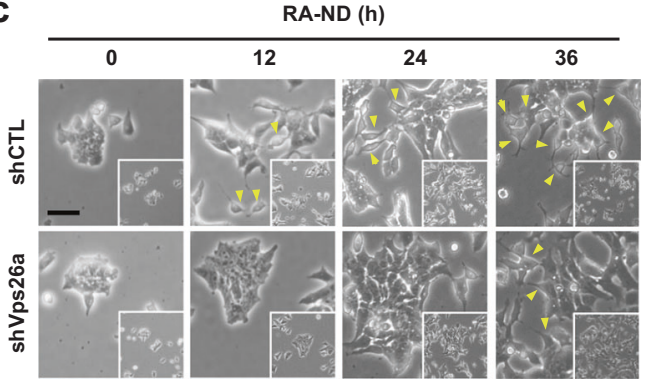

e
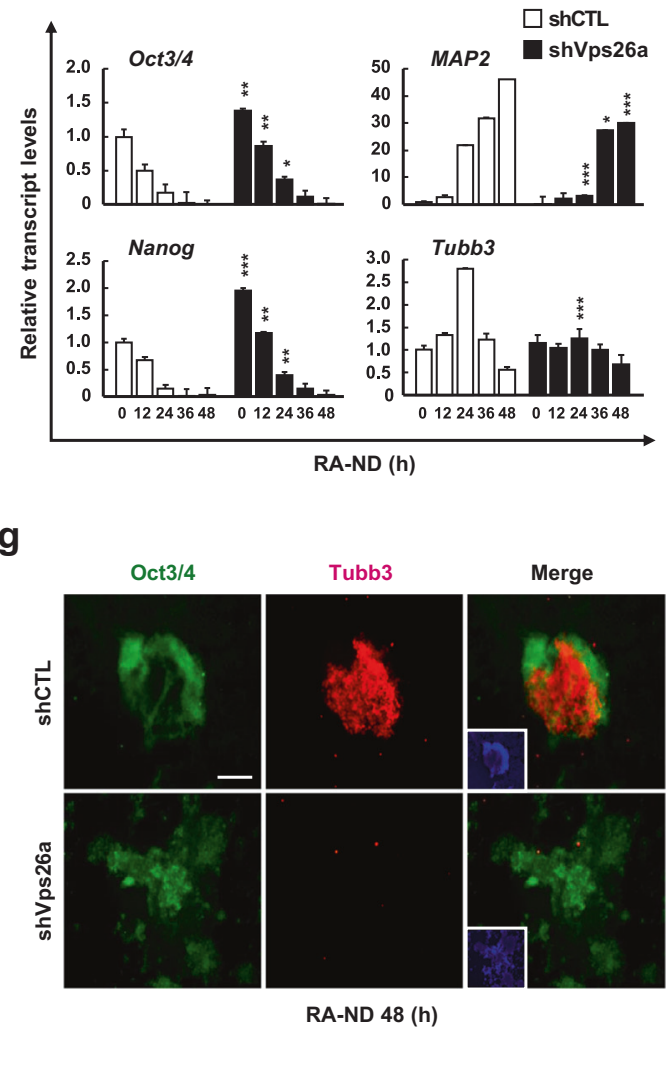

RA-ND 48 (h) g f-i

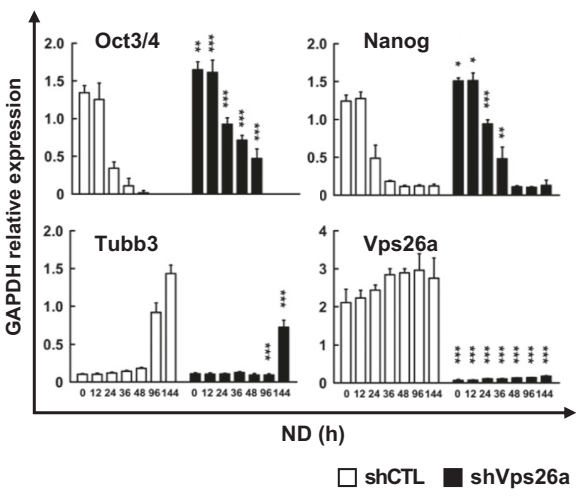

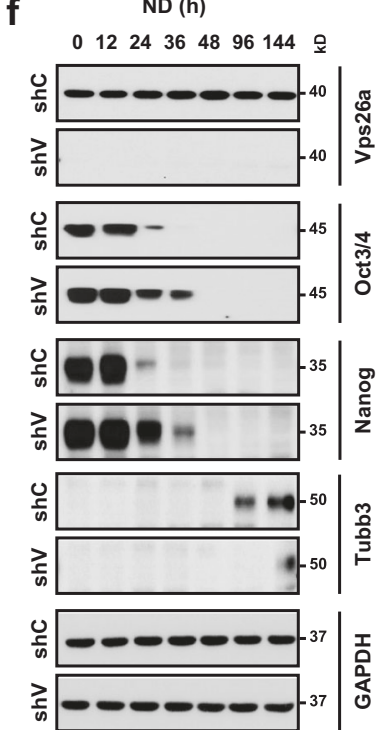

Fig. 2 Vps26a knockdown prolongs the maintenance of stemness under ND conditions in P19 ECCs. a, b The effect of Vps26a knockdown on the expression of ESC stemness genes were determined by semi-qPCR (a) and western blot (b) analyses of Oct3/4 and Nanog using shCTL- and shVps26a-ECCs. c Morphological changes of shCTL (shC)- and shVps26a (shV)-ECCs during retinoic acid-induced neurogenesis (RA-ND) for the indicated time periods. The yellow arrowheads indicate cells with differentiated morphologies. d-f Expression kinetics of ESC stemness and neuronal markers examined by semi-qPCR (d), qPCR (e), and western blotting (f)

following treatment with $\mathrm{N}$-acetyl L-cysteine (NAC), a chemical ROS scavenger, restored the immunoreactivity of analyses using shC- and shV-ECCs differentiated for the indicated time periods. Error bars indicate the means $\pm \mathrm{SD}(n=3) .{ }^{*} P<0.05$; ${ }^{* *} P<0.01 ;{ }^{* * *} P<0.001$ compared with the shC-ECCs from each day during ND. $\mathbf{f}-\mathbf{i}$ Immunoblotting quantification of (f). Quantification of the fluorescence intensity was performed using ImageJ software $(n=3$ for each group). Error bars are \pm SD. ${ }^{*} P<0.05 ;{ }^{* *} P<0.01$; ${ }^{* * *} P<$ 0.001 vs. shCTL ND 0. g Double-label immunocytochemical analysis of Oct3/4 (green) and Tubb3 (red) during ND for $48 \mathrm{~h}$. DAPI staining data are shown as insets to the merged images. Scale bar, $50 \mu \mathrm{m}$

Oct3/4 and Nanog in differentiating WT ESCs to the levels of Vps26a ${ }^{-1-}$ cells (Fig. 4c-i). 
a

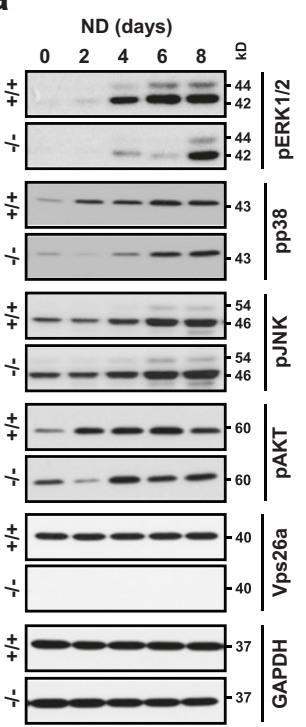

b

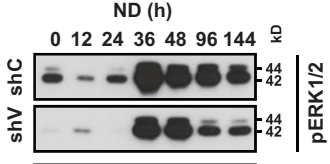

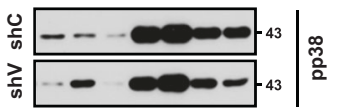

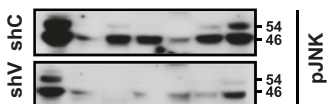

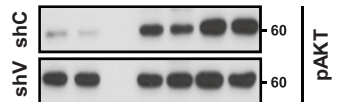

O- - - - - 40

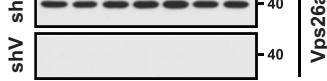

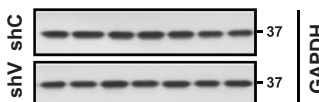

a-i

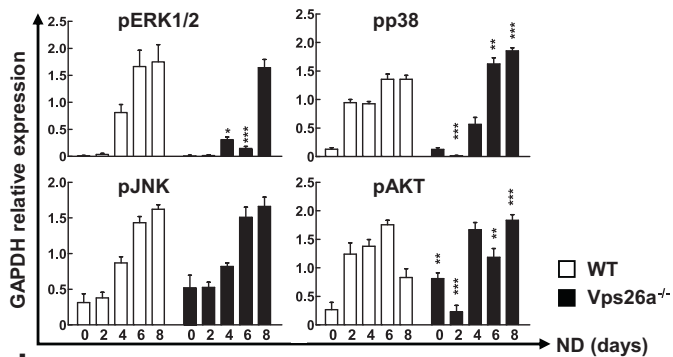

b-i

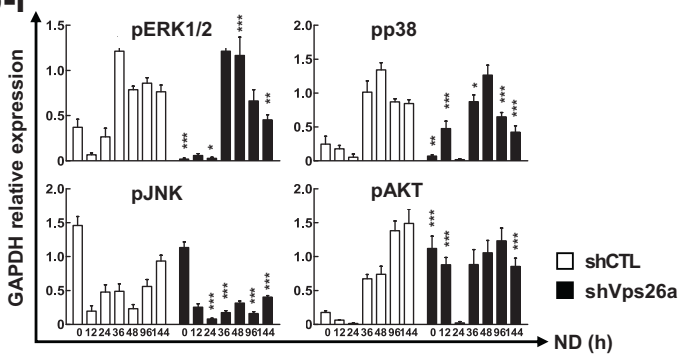

C

Inhibitors

Inhibitors

- PD U LY SB SP $\stackrel{?}{2}$

- PD U LY SB SP
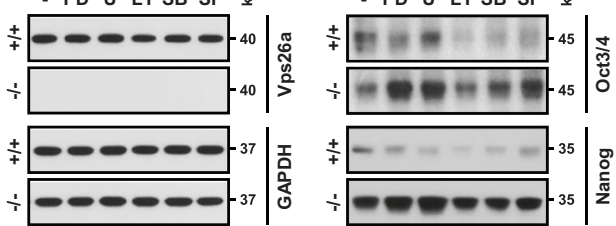

d

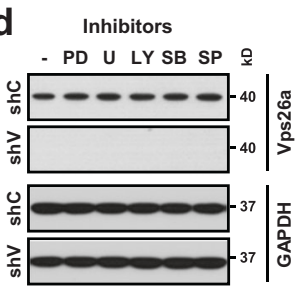

Inhibitors

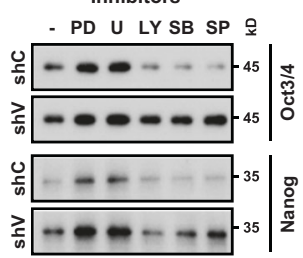

d-i

c-i

Oct3/4

Nanog
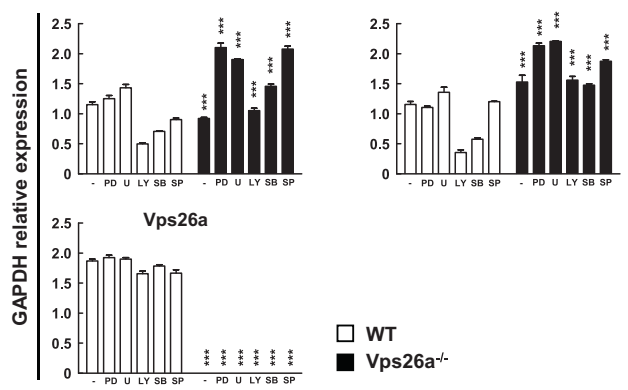

$\square$ WT

Vps26a ${ }^{-/-}$
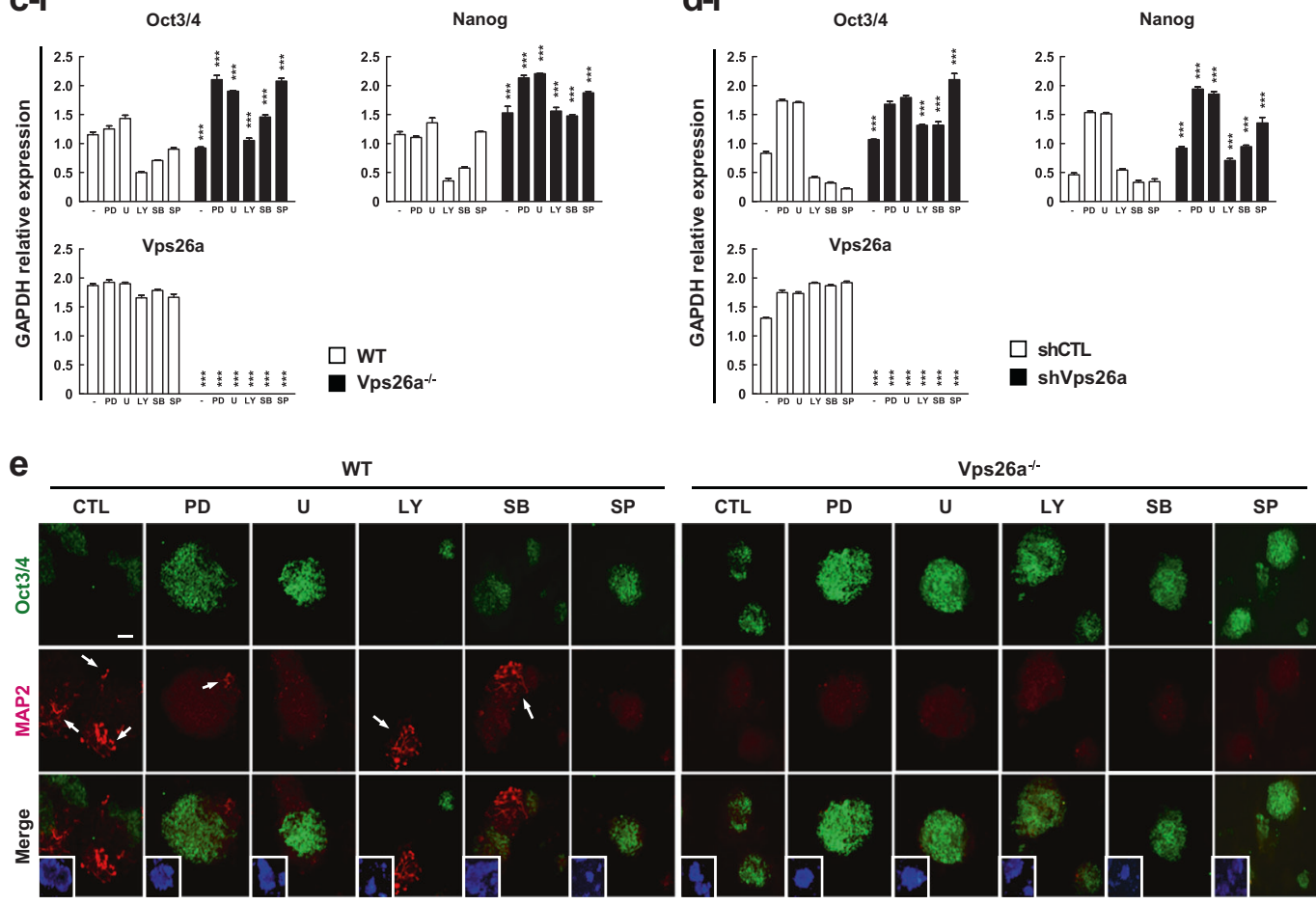

To identify the source of ROS during neural differentiation from ESCs, the expression levels of Nox family proteins, which are representative cellular ROS-generating

enzymes, were investigated by semi-qPCR and qPCR analyses using differentiating shCTL ECCs. Except for Nox2, most of the Nox enzymes increased during neural 
Fig. 3 Vps26a-mediated neurogenesis depends on the ERK1/2 cascade. a, b The effects of deficiency or knockdown of Vps26a on pERK1/2, pp38 MAPK, pJNK, and pAKT levels were determined by western blotting analysis using ESCs (a) or ECCs (b), respectively, differentiated for the indicated time periods. a-i, b-i Immunoblotting quantification of (a) and (b). Western blot signals were quantified and the intensity of phosphorylated protein and the total protein normalized to the loading control GAPDH are presented ( $n=3$ for each group). Error bars are \pm SD. ${ }^{*} P<0.05 ;{ }^{* *} P<0.01 ;{ }^{* * *} P<0.001$ vs. $+/+$ ND 0 (a-i) and shCTL ND 0 (b-i). c, d The effect of signaling inhibitors for ERK1/2 (PD98059, PD; U0126, U), p38 MAPK (SB203580, SB), JNK (SP600125, SP), and AKT (LY294002, LY) on expression of ESC stemness markers was examined by western blot analysis of Oct3/4 and Nanog using WT (+/+) and Vps26a $a^{-/-}(-/-)$ESCs differentiated for 6 days (c) and shCTL (shC)- and shVps26a (shV)-ECCs differentiated for $144 \mathrm{~h}$ (d). c-i, d-i Immunoblotting quantification of (c) and (d). Western blot signals were quantified and the intensity of phosphorylated protein and the total protein normalized to the loading control GAPDH are presented ( $n=3$ for each group). Error bars are \pm SD. ${ }^{* * *} P<0.001$ vs. +/+ ND 6 (c-i) and shCTL ND 6 (d-i). e Doublelabel immunocytochemical analysis of Oct3/4 (green) and MAP2 (red) using WT and Vps26a $a^{-/-}$ESCs differentiated in the presence or absence of the indicated signaling inhibitors for 6 days. DAPI staining data are shown as insets to the merged images. Scale bar, $50 \mu \mathrm{m}$

differentiation from ECCs (Fig. 5a, b). In particular, gradual upregulation of Nox3 and Nox4 was significantly reduced by knockdown of Vps26a in shCTL ECCs (Fig. 5a, b). Consistent with this observation, whole-mount immunohistochemistry revealed that Nox4 expression was also markedly diminished in $V p s 26 a^{-/-}$embryos compared with WT embryos at embryonic day 10.5 (E10.5) (Fig. 5c). Moreover, immunocytochemistry revealed strong Nox4 immunoreactivity in differentiating ESCs, which was greatly reduced in $V p s 26 a^{-/-}$cells compared with WT cells (Fig. 5d). Furthermore, Vps $26 a^{-/}$ESC-derived teratomas suppressed differentiation and sustained Oct3/4 expression levels, whereas WT ESC-derived teratomas upregulated the expression of Tubb3 and Nox4 (Fig. 5e). We found that overexpression of Nox4 increased MAP2-positive cells from shVps26a ECCs, while decreased in Oct3/4-positive cells. These results demonstrate that neurogenesis capacity can be restored by regulating the gene of Nox4, which is reduced to Vps26a deficiency (Supplementary Fig. 9).

\section{Vps26a interacts with Nox4}

To identify a possible interaction between Vps26a and Nox4, we first examined whether both proteins colocalize in embryos. Most Vps26a-positive areas were precisely colocalized with areas of Nox4 in Tubb3-positive neuronal cells at E10.5 by whole-mount immunohistochemistry (Fig. 6a). Moreover, Vps26a and Nox4 exhibited a specific interaction in differentiating WT cells but not in Vps $26 \mathrm{a}^{-/}$cells, as revealed by immunoprecipitation experiments in undifferentiated and differentiating ESCs, and displayed a stronger interaction, along with neuronal differentiation, accompanied by increased protein levels of Nox4 and Vps26a (Fig. 6b). Consistent with these results, HA-Nox4C coprecipitated with purified His-tagged Vps26a (Fig. 6c) and glutathione S-transferase (GST)-Vps26a (Fig. 6d) in the binding assay. To determine the region of $\mathrm{Vps} 26 \mathrm{a}$ that interacts with Nox4, a series of Vps26a-truncated proteins tagged with GST were expressed, and their abilities to interact with HA-Nox4C were tested using a GST-pulldown assay. Nox4 interacted with the N-terminal Vps26a (amino acids; aa 1-148) protein (Fig. 6e, lane 4), whereas Nox4 failed to interact with the C-terminal Vps26a (aa 149245 and 246-360) protein (Fig. 6e, lanes 5 and 6).

\section{Vps26a-mediated regulation of the stemness/ differentiation transition depends on the synergy between Nox, ROS, and ERK1/2}

To define the involvement of Nox-generated ROS in the Vps26a-dependent transition of stemness/differentiation during neurogenesis, WT and Vps $26 a^{-/}$ESCs were differentiated in the presence or absence of NAC or diphenyleneiodonium (DPI), a potent Nox inhibitor, and flow cytometry was performed to measure ROS, along with qPCR and immunocytochemistry to investigate the expression of stemness/differentiation markers. Similar to treatment with the antioxidant NAC, supplementation of DPI successfully decreased ROS generation in undifferentiated and differentiating ESCs, regardless of the Vps26a genotype (Fig. 7a). Under reduced ROS following treatment with either NAC or DPI, Oct3/4 and Nanog transcription levels in WT cells were restored to the levels of $V p s 26 a^{-/}$ESCs, whereas the increase in $T u b b 3$ expression was significantly reduced in WT cells (Fig. 7b). Consistent with these findings, Oct3/4 and Nanog immunoreactivity was enhanced by treatment with either NAC or DPI, with intensities comparable with those of $V p s 26 a^{-/}$ESCs (Fig. 7c-i). When Nox4 was reduced using siRNA, Oct3/4 and Nanog were expressed higher than siCTL, and MAP2 was remarkably decreased in ECCs with Nox4 siRNA. These results are consistent with previous findings that increased Nox4mediated ROS production improves neural progenitor cell differentiation [22, 23] (Supplementary Fig. 10). Taken together, Nox4 seems to play an important role in neuronal differentiation through interaction with Vps26a.

To determine whether ERK1/2 activation is required for Nox-generated ROS, western blot analysis of three MAPKs and AKT was performed using Vps26a ${ }^{-/}$ESCs and shVps26a-ECCs cultured or differentiated in the presence or absence of hydrogen peroxide, NAC, or DPI. Of the kinases investigated, pERK1/2 levels were markedly reduced in $V p s 26 a^{-1}$ ESCs compared with WT cells, although the levels responded to hydrogen peroxide in both genotypes (Fig. 8a, b). Additionally, treatment with NAC or DPI greatly 
a

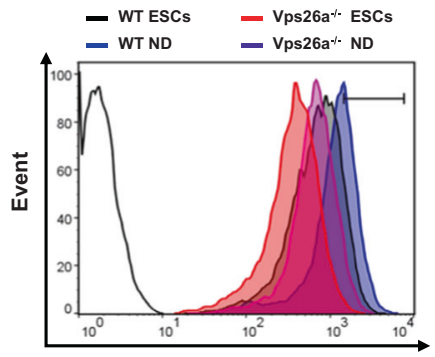

CM- $\mathrm{H}_{2}$ DCFDA fluorescence

b

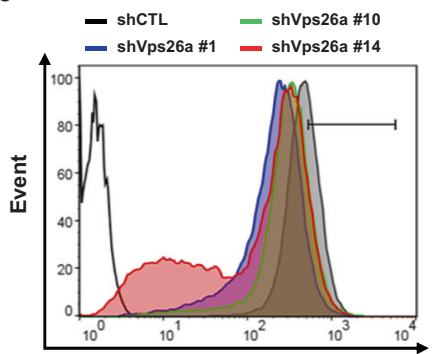

C

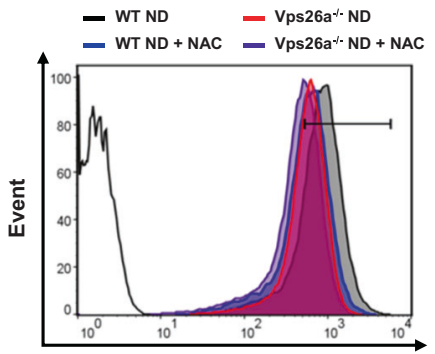

CM- ${ }_{2}$ DCFDA fluorescence
CM- ${ }_{2}$ DCFDA fluorescence
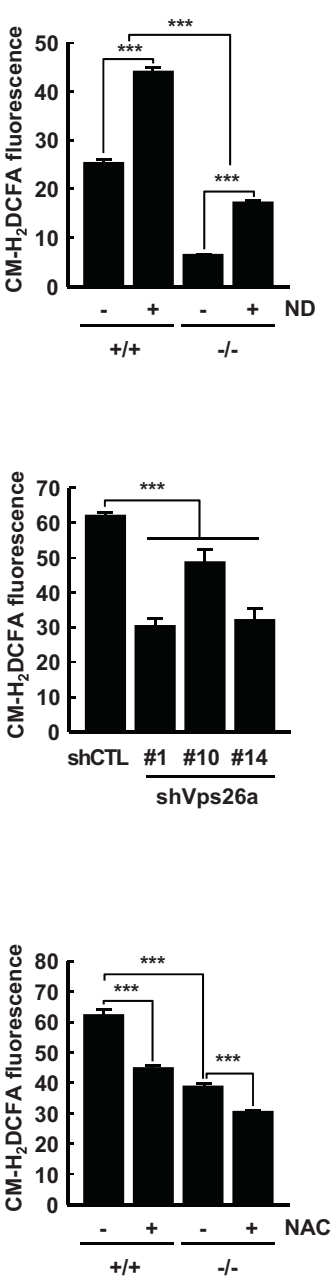

Fig. $4 \mathrm{Vps} 26 \mathrm{a}$ is required for increased ROS leading to ESC-mediated neurogenesis. a, b The effect of Vps26a deficiency (a) or knockdown (b) on ROS generation was determined by flow cytometry using WT and $V p s 26 a^{-/-}$ESCs (a) and shCTL- and shVps26a-ECCs (\#1, \#10, and \#14) (b) differentiated for 6 days and $48 \mathrm{~h}$, respectively. Unstained cells (black lines) were used as a negative control. The data are representative of at least three independent experiments and presented as means $\pm \mathrm{SD}(n=3) .{ }^{* * *} P<0.001$. c WT and $V p s 26 a^{-1-}$ ESCs were differentiated in the presence or absence of $2.5 \mathrm{mM}$ NAC for 6 days, and ROS levels were measured by flow cytometry. The data are

decreased the intensity of the pERK1/2 and in WT cells, comparable with the levels in the Vps26a ${ }^{-/-}$groups (Fig. 8c), showing a similar pattern to the western blot analysis using shCon- and shVps26a-ECCs (Fig. 8d). pERK1/2 immunoreactivity was markedly reduced by supplementation with either NAC or DPI during neural differentiation of WT cells (Fig. 8e), as demonstrated in the western blot analysis. More interestingly, the levels of ROS (Fig. 8f, g) and Nox4 (Fig. 8h) were markedly reduced following treatment with PD98059, indicating crosstalk feedback between Nox4/ROS and ERK1/ 2 during neurogenesis from ESCs. d
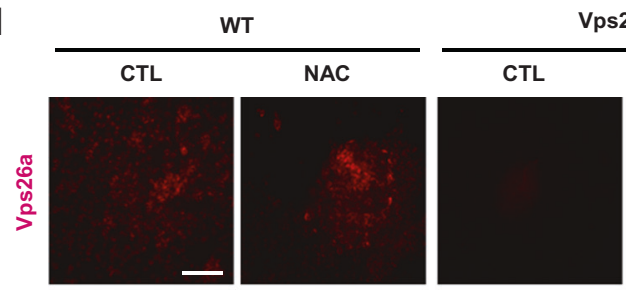

Vps26a-
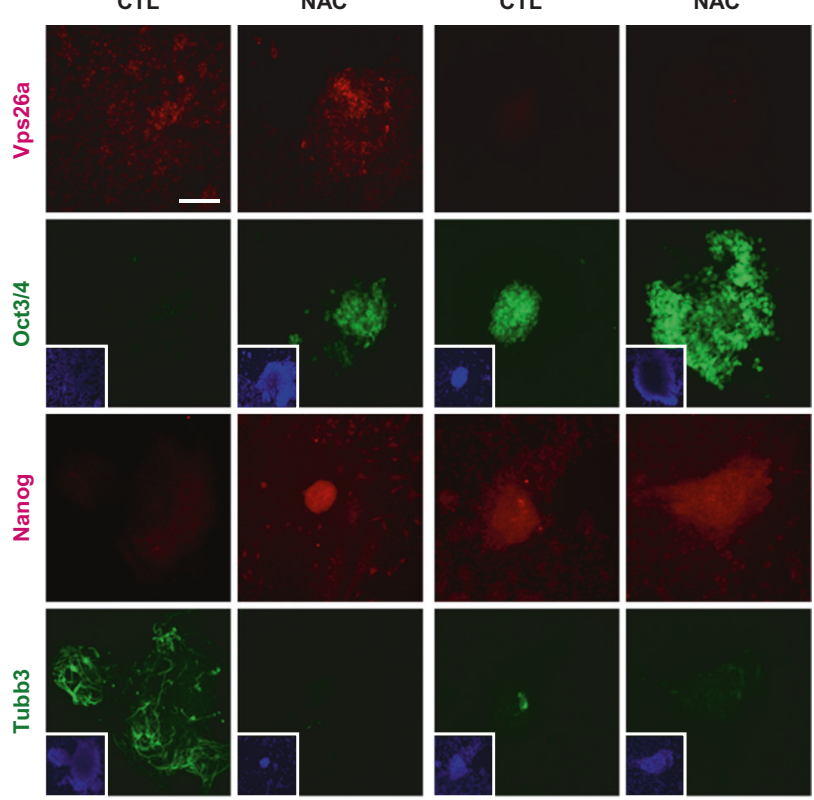

d-i

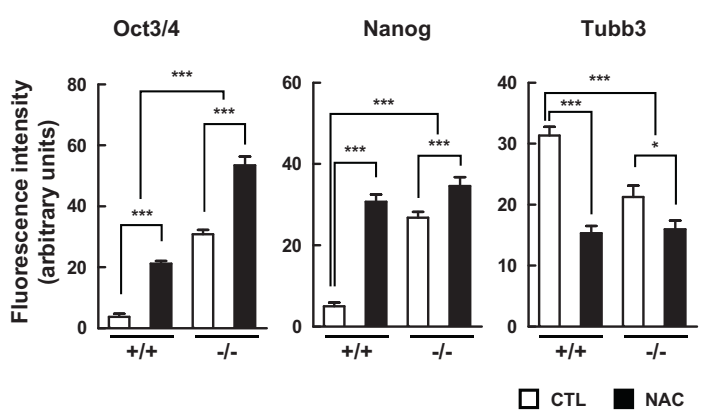

representative of at least three independent experiments and presented as means $\pm \mathrm{SD}(n=3)$. ${ }^{* * *} P<0.001$. d Double-label immunocytochemical analysis of Vps26a (red), Oct3/4 (green), Nanog (red), and Tubb3 (green) using WT and Vps26a $a^{-/}$ESCs differentiated in the presence or absence of $2.5 \mathrm{mM}$ NAC for 3 days. DAPI staining data are shown as insets to the Oct3/4 and Tubb3 images. Scale bar, $50 \mu \mathrm{m}$. $\mathbf{d}-\mathbf{i}$ immunofluorescence quantification of $(\mathbf{d})$. Quantitative analysis of the fluorescence intensity performed using ImageJ software $(n=3)$. Error bars are \pm SD. ${ }^{*} P<0.05 ;{ }^{* * *} P<0.001$ vs. WT ND 6 CTL

\section{Discussion}

The insertion mutation of Vps26a in mice leads to embryonic lethality due to failure in early postimplantation development [10]. To date, few studies have investigated the mechanism(s) linking developmental abnormalities in $V p s 26 a^{-/}$fetuses. To address this, Vps $26 a^{-/}$ESCs were generated and used as an in vitro model of differentiation. Neurogenesis analyses of these cell lines revealed that Vps26a actively participates in regulation of the stemness/ differentiation transition via cooperation with redox 

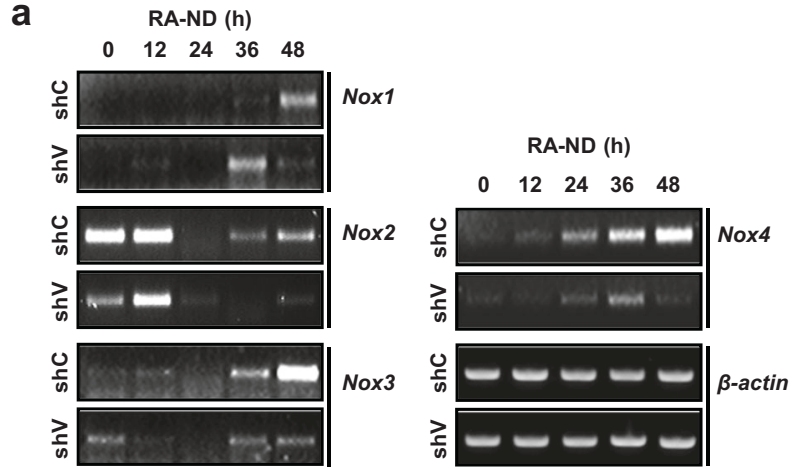

b

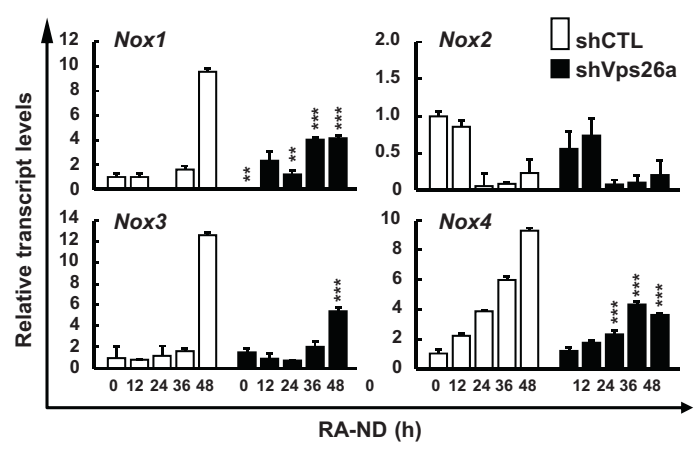

e

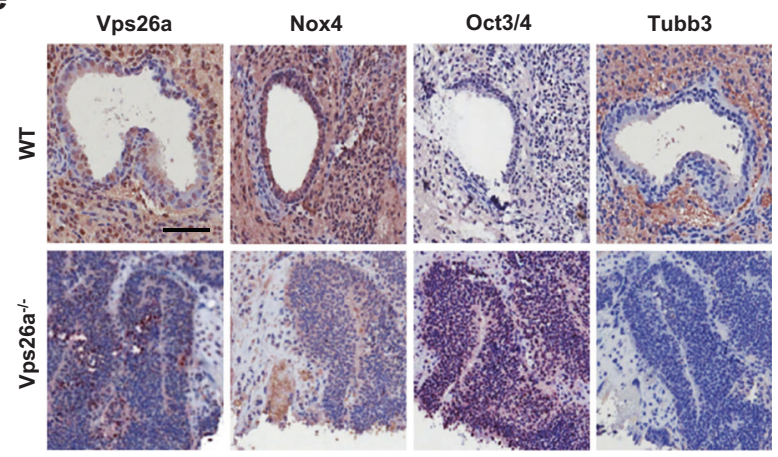

Fig. 5 Vps26a involves Nox expression during neurogenesis. a, b The effect of Vps26a deficiency and knockdown on the expression of the Nox family was determined by semi-qPCR (a) and qPCR (b) analyses using shCTL (shC)- and shVps26a (shV)-ECCs, differentiated for the indicated time periods. Error bars indicate the means $\pm \operatorname{SD}(n=3)$. ${ }^{* *} P$ $<0.01 ;{ }^{* * *} P<0.001$ compared with shCTL-ECCs each day. c Overview of Vps26a and Nox4 immunostaining of a sagittal section of WT

signaling. Furthermore, these findings represent the first demonstration of an association between the retromer complex and ROS.

Researchers have investigated redox-sensitive mechanisms regulating the behavior of ESCs during differentiation, including the involvement of antioxidant enzymes. However, the expression kinetics of antioxidant enzymes does not always correlate with intracellular ROS levels. Following application of neural differentiation stimuli, the
C

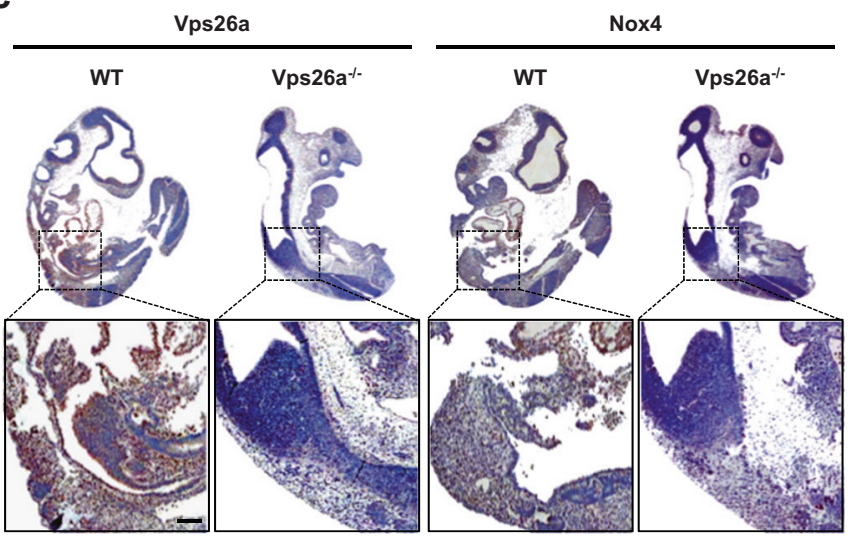

d

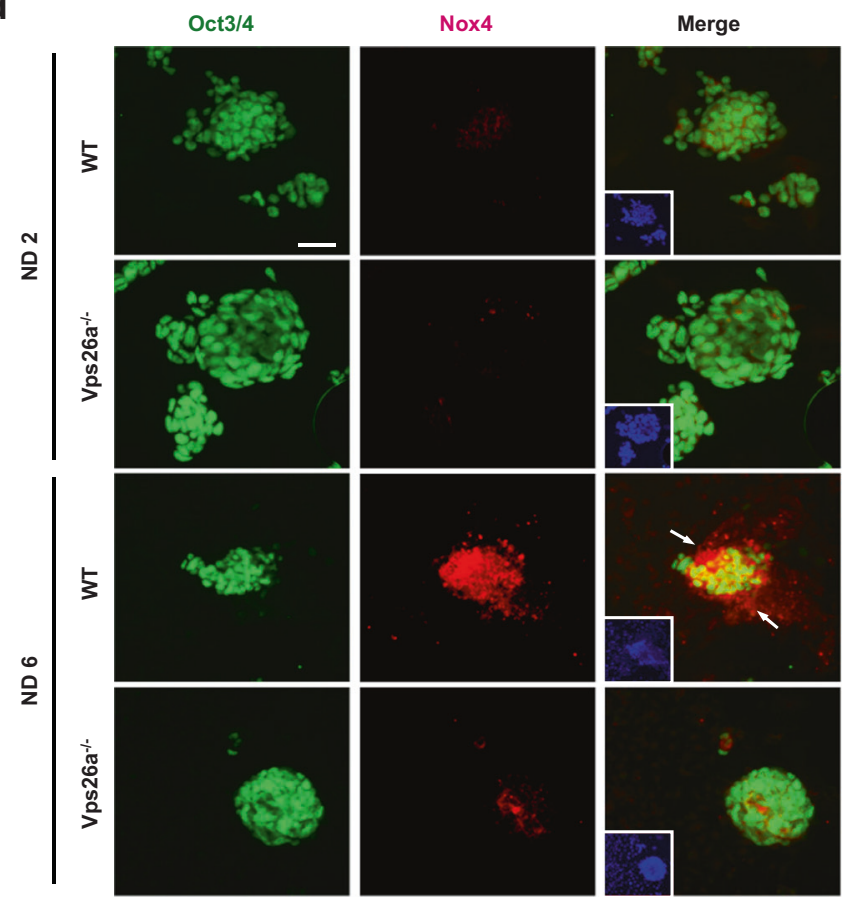

and $V p s 26 a^{--}$mouse embryos (E10.5) counterstained lightly with hematoxylin. Scale bar, $200 \mu \mathrm{m}$. d Double-label immunocytochemical analysis of Oct3/4 and Nox4 using WT and Vps26a ${ }^{-/}$ESCs differentiated for 6 days. DAPI staining data are shown as insets to the merged images. Scale bar, $50 \mu \mathrm{m}$. e Expression of Nox4, Oct3/4, and Nox4 in WT and Vps $26 a^{-/-}$teratomas. Scale bar, $100 \mu \mathrm{m}$

antioxidant enzymes Prx I and Prx II showed opposing expression levels due to the intercellular distribution of Prx I and Prx II in ESCs and neural-lineage cells, respectively [19]. Consistent with this finding, the present study also identified opposing expression kinetics of Prx I and Prx II during neurogenesis from ESCs, which were severely retarded in differentiating $V p s 26 a^{-/}$ESCs compared with WT cells (Supplementary Fig. 11). These results suggest that neurogenesis from ESCs requires the stepwise 
a

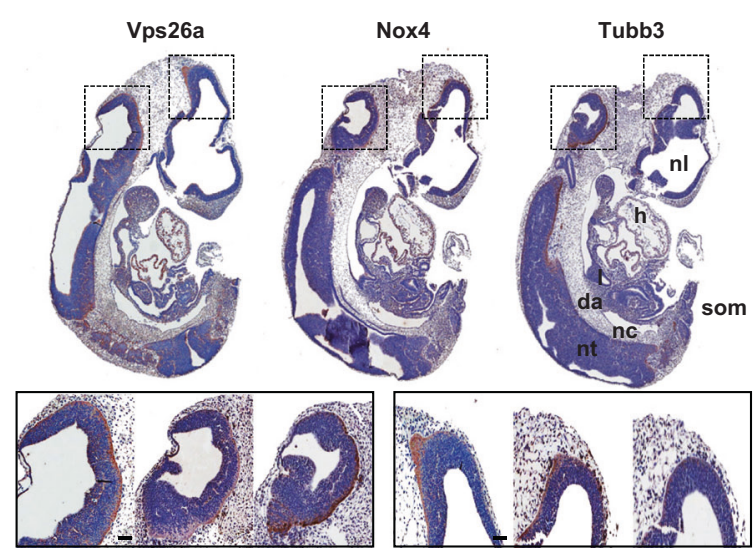

c

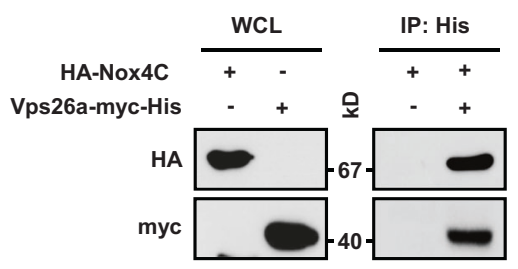

e
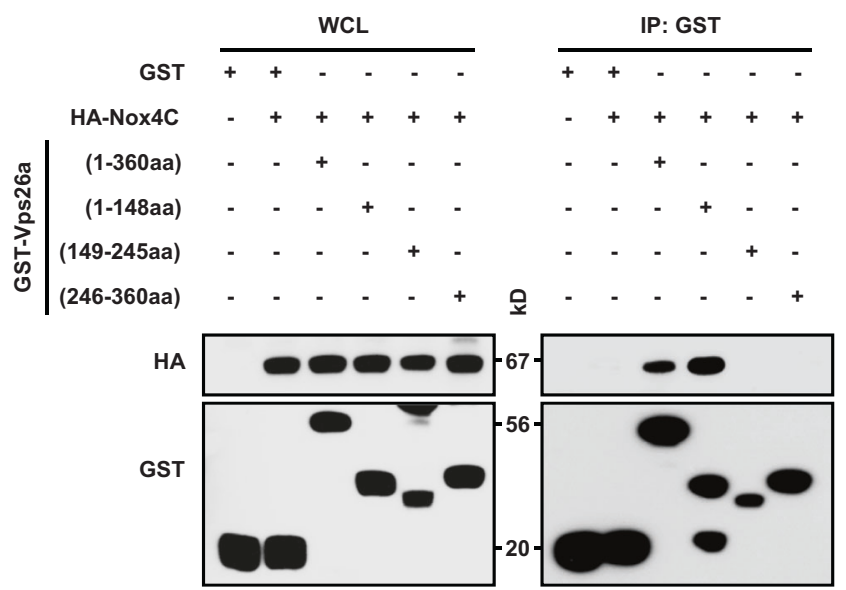

b

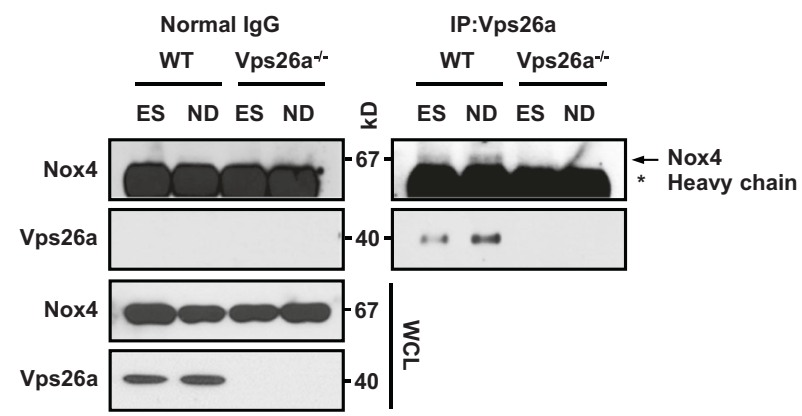

d

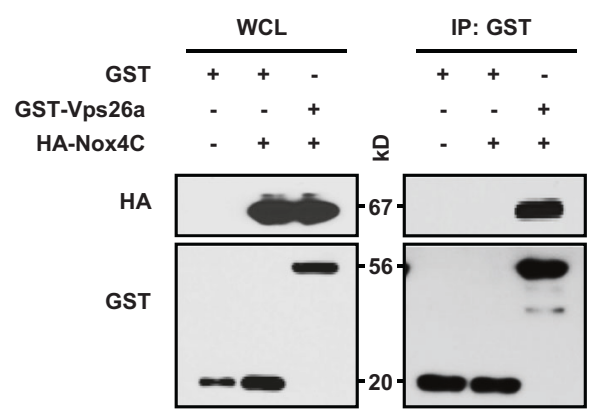

Fig. 6 Identification of the interaction between Vps26a and Nox4. a Serial sagittal sections of WT embryos were immunostained for Vps26a, Nox4, and Tubb3 and counterstained lightly with hematoxylin at E10.5. da, dorsal aorta; 1, lung; h, heart; nc, notochord; nl, neural lumen; nt, neural tube; som, somite. b Vps26a immunoprecipitation followed by anti-Vps26a and -Nox4 immunoblots using lysates obtained from WT $(+/+)$ and $V p s 26 a^{-/-}(-/-)$ESCs during neural differentiation for 0 or 6 days. IgG was used as an immunoprecipitation control. c GST-tagged Vps26a was subjected to a pulldown assay with the lysates of HEK293 cells transfected with HANox4C (C-terminal region, 249-574 aa)-expressing plasmid. Immunoblot analysis with anti-HA antibody is shown at the top. Equal

and cell-specific involvement of antioxidants without greatly affecting total intracellular ROS levels. The current aim is to define the roles of specific antioxidant enzymes in their preferentially expressed cells during differentiation. loading of the GST proteins assessed by GST antibody is shown at the bottom. GST was used as a negative control. d Cell lysates from HEK293 cells transfected with HA-Nox4C were mixed with purified His-tagged Vps26a. Samples were immunoblotted with the HA tag antibody. Levels of input protein are shown by immunoblotting with the HA- and Myc-tag antibodies. e Expression constructs for GSTtagged serial deletion mutants of Vps26a and HA-Nox4C were coexpressed in HEK293 cells. Immunoblot analysis with anti-HA antibody is shown at the top. Equal loading of the GST proteins assessed by GST antibody is shown at the bottom. GST was used as a negative control

The retromer complex has been implicated in degenerative neurological diseases, including Alzheimer's disease (AD) and Parkinson's disease (PD) [7, 24, 25]. Null mutations in Vps26 and Vps35 in mice accelerate the production of $\beta$ amyloid $(A \beta)$, a cleaved product of the amyloid precursor 


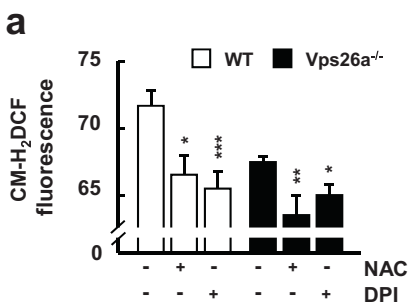

C

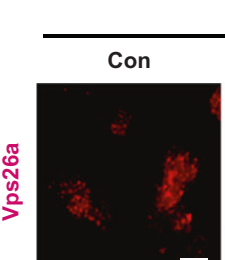

WT

ps26a

b
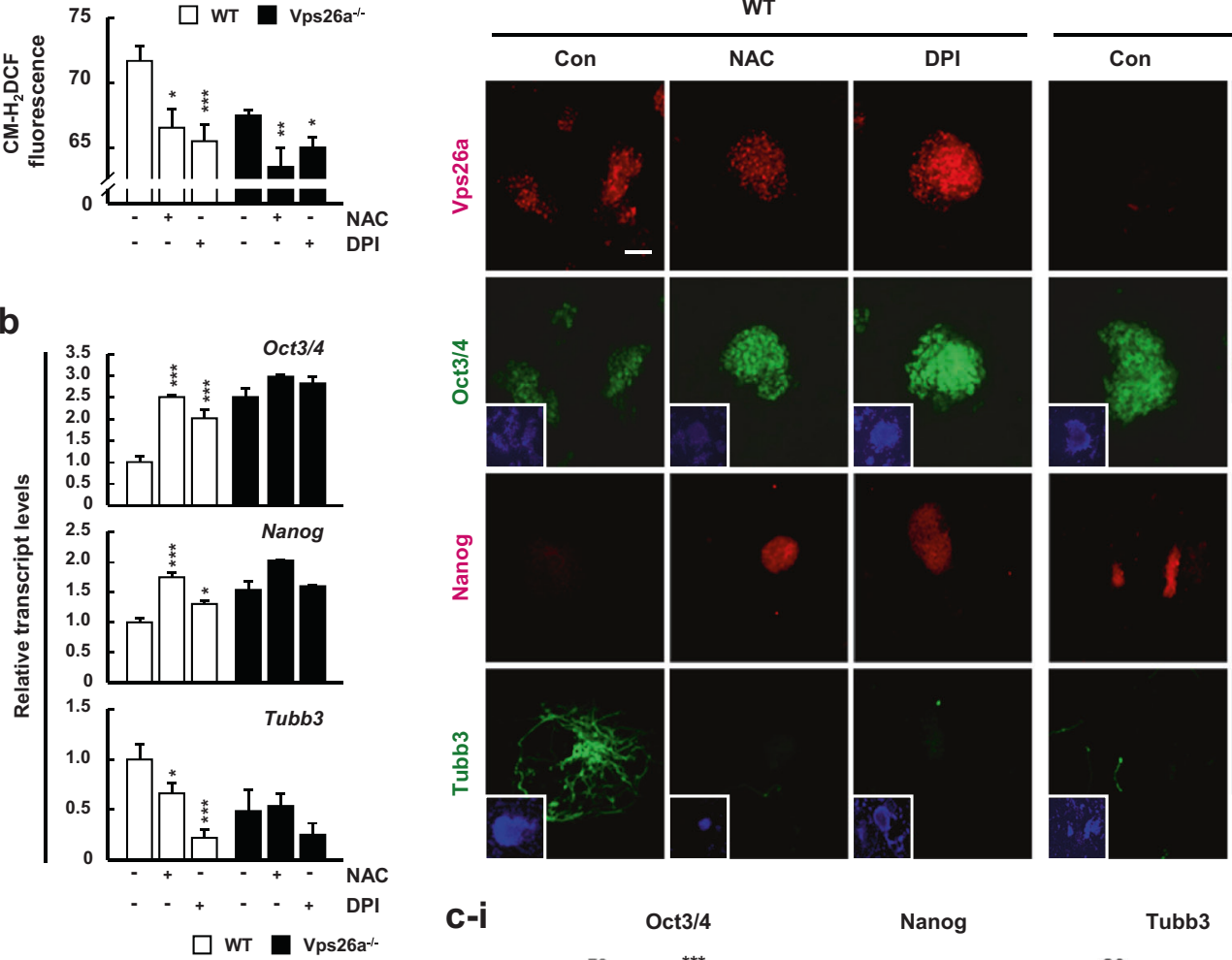

NAC

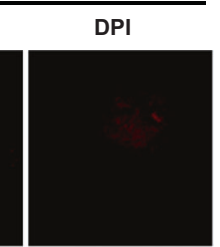

葛
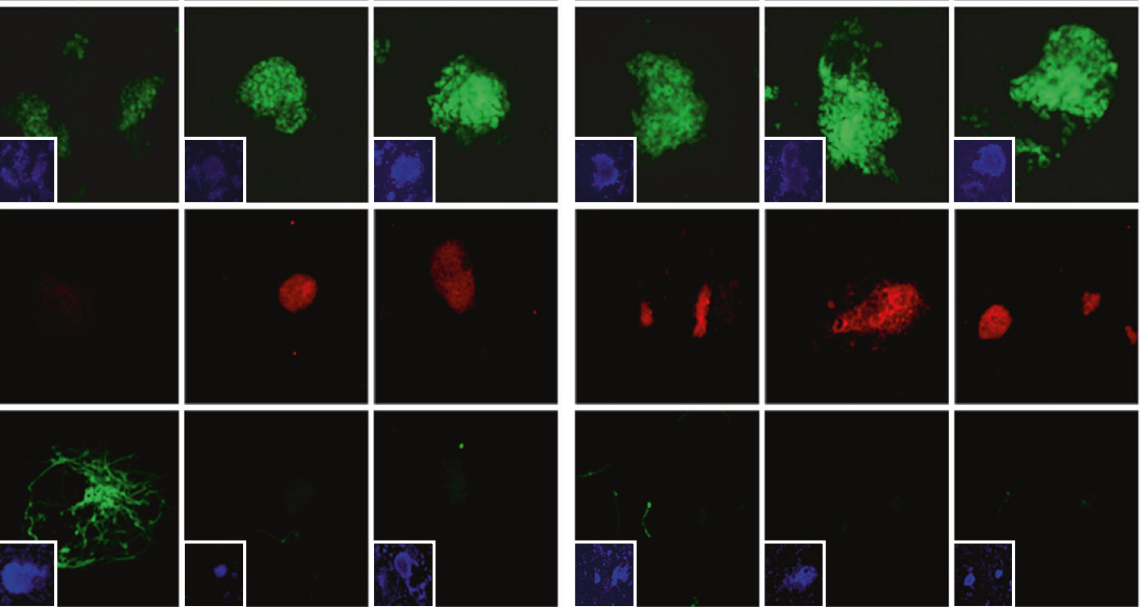

C-I
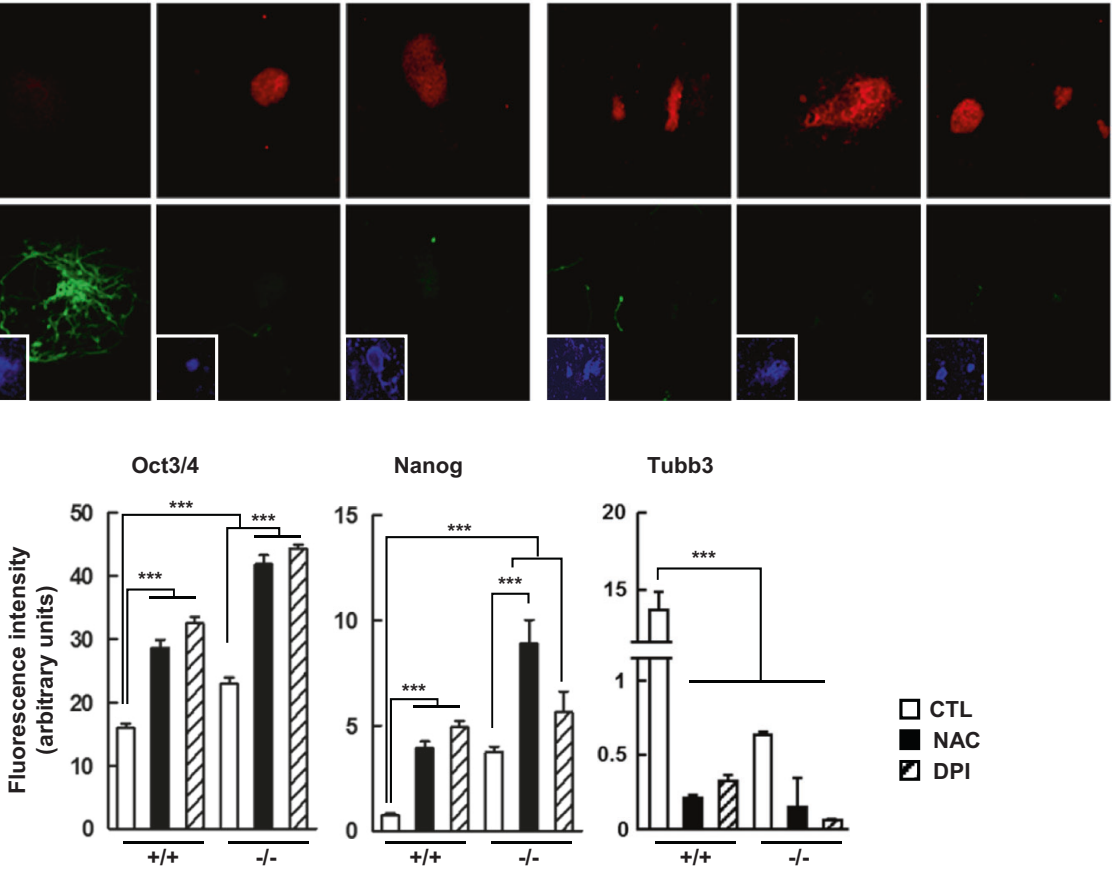

$\square$ WT $\square$ Vps26a-

Fig. 7 Nox4-generated ROS lead to a loss of stemness and subsequent neurogenesis from ESCs. a, b The effects of antioxidant and Nox inhibitor treatments on ROS generation (a) and ESC stemness transcription levels (b) were examined by flow cytometry using WT and $V p s 26 a^{-1-}$ ESCs differentiated in the presence or absence of $2.5 \mathrm{mM}$ NAC or $10 \mu \mathrm{M}$ DPI for 6 days. Error bars indicate the means \pm SD $(n=3) .{ }^{*} P<0.05 ;{ }^{* * *} P<0.01 ;{ }^{* * * *} P<0.001$ compared with no treatment. c Double-label immunocytochemical analysis of Vps26a (red),
Oct3/4 (green), Nanog (red), and Tubb3 (green) using WT and $V p s 26 a^{-\gamma}$ ESCs differentiated in the presence or absence of $20 \mathrm{mM}$ $\mathrm{NAC}$ or $2.5 \mu \mathrm{M}$ DPI for 6 days. DAPI staining data are shown as insets to the Oct3/4 and Tubb3 images. Scale bar, $50 \mu \mathrm{m}$. c-i Immunofluorescence quantification of c. Quantification of the fluorescence intensity was performed using ImageJ software $(n=3)$. Error bars are \pm SD. ${ }^{*} P<0.05 ;{ }^{* * * *} P<0.001$ vs. WT ND 6 CTL

activity; [32] this is indicative of a close association between the retromer complex and neurodegenerative pathogenesis. In general, neurodegenerative disorders frequently entail oxidative stress or excessive ROS levels. ROS overloads are frequently detected in $\mathrm{AD}$ and PD models of cells or animals $[33,34]$, and altered ROS levels are generally accepted as a marker of neurological disease [35]. Nevertheless, the association between the retromer complex and redox status has not yet been clarified. Our findings identified a relationship between Vps26a and ROS that could have a marked impact on retromer complex studies in relation to incurable 
a

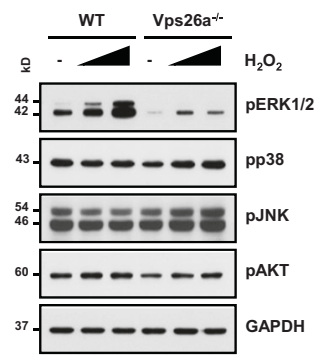

a-i

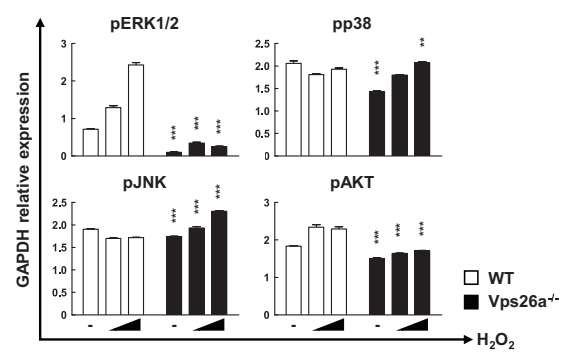

C

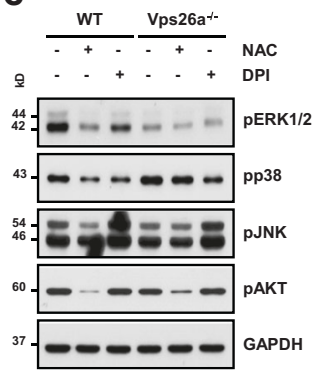

C-i

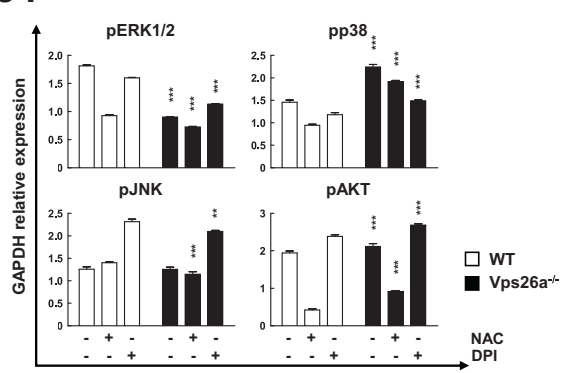

e

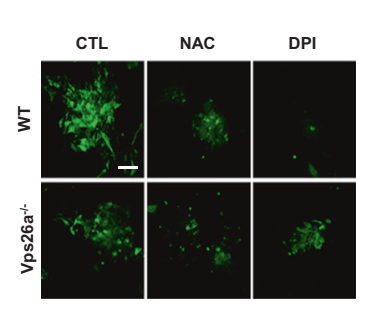

f

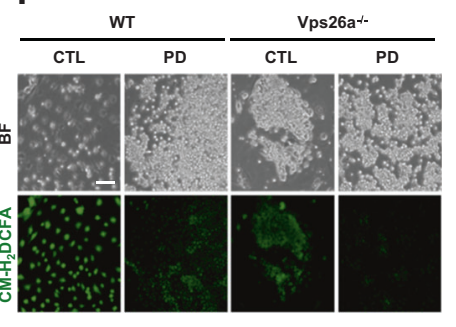

b

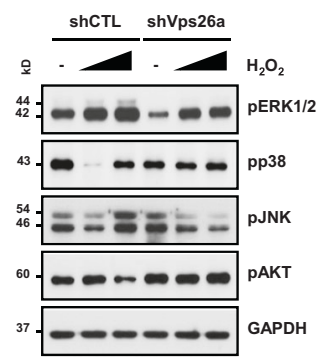

b-i

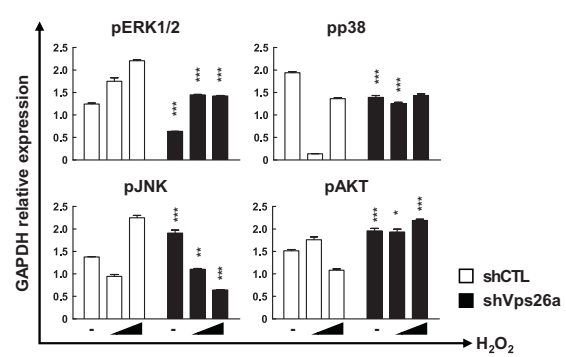

d

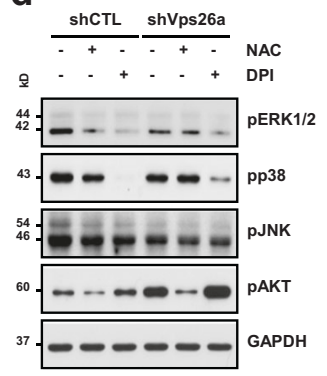

d-i

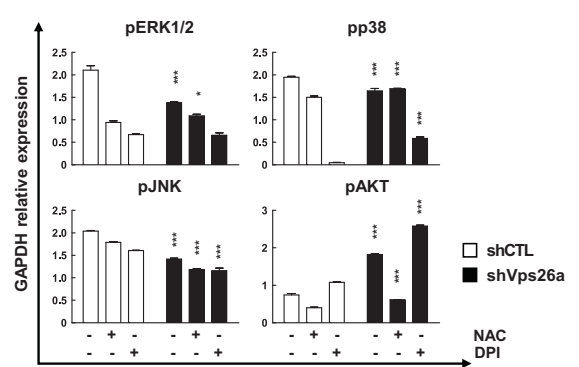

g

h
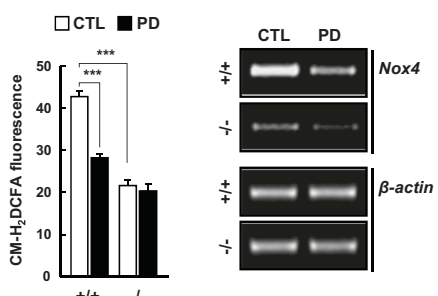

degenerative disorders. We aim to clarify the molecular mechanism(s) underlying the involvement of ROS in regulating the retromer complex.
Many studies have demonstrated that MAPKs, such as ERK1/2, p38 MAPK, and JNK play an important role in differentiation processes [18]. Among the three MAPKs, 
Fig. 8 Vps26a-mediated neurogenesis depends on cooperation between Nox, ROS, and the ERK1/2 cascade. a, b The effects of oxidant treatment on modulation of pERK1/2, pp38 MAPK, pJNK, and pAKT levels were determined by western blot analysis using WT $(+/+)$ and Vps26a $a^{-/}$(-/-) ESCs or shCTL (shC)- and shVps26a (shV)ECCs (b) treated with increasing concentrations of hydrogen peroxide $(50$ and $100 \mu \mathrm{M})$, respectively, for $10 \mathrm{~min}$ at the end of neural differentiation (6 days). $\mathbf{a}-\mathbf{i}, \mathbf{b}-\mathbf{i}$ Immunoblotting quantification of (a) and (b). Western blot signals were quantified and the intensity of phosphorylated protein and the total protein normalized to the loading control GAPDH are presented ( $n=3$ for each group). Error bars are \pm SD. ${ }^{* * *} P<0.01 ;{ }^{* * * *} P<0.001$ vs. +/+ ND $6(\mathbf{a}-\mathbf{i})$ and shCTL ND 6 (bi). c, d The effects of antioxidant- and Nox inhibitor treatments on modulation of pERK1/2, pp38 MAPK, pJNK, and pAKT levels were determined by western blot analysis using WT and Vps26a $a^{-/-}$ESCs (c) or shCTL- and shVps26a-ECCs (d) cultured in the presence or absence of $20 \mathrm{mM}$ NAC or $2.5 \mu \mathrm{M}$ DPI for $6 \mathrm{~h}$ at the end of neural differentiation (6 days and $144 \mathrm{~h}$, respectively). c-i, d-i Immunoblotting quantification of (c) and (d). Western blot signals were quantified and the intensity of phosphorylated protein and the total protein normalized to the loading control GAPDH are presented ( $n=3$ for each group). Error bars are \pm SD. ${ }^{* * *} P<0.001$ vs. $+/+$ ND $6(\mathbf{c}-\mathbf{i})$ and shCTL ND 6 $(\mathbf{d}-\mathbf{i})$. e Immunocytochemical analysis of pERK1/2 using WT and $V p s 26 a^{-/-}$ESCs differentiated in the presence or absence of $20 \mathrm{mM}$ NAC or $2.5 \mu \mathrm{M}$ DPI for 6 days. Scale bar, $50 \mu \mathrm{m}$. f, $\mathbf{g}$ The effect of ERK1/2 inhibitor treatment on ROS generation examined by fluorescence microscopy (f) or flow cytometry (g) using $+/+$ and -/- ESCs differentiated in the presence or absence of PD98059 for 3 days. Error bars indicate the means $\pm \operatorname{SD}(n=3) .{ }^{* * * *} P<0.001$. h The effect of ERK inhibitor treatment on the expression of Nox 4 determined by semi-qPCR analysis using $+/+$ and $-/$ - ESCs differentiated in the presence or absence of PD98059 for 6 days

ERK1/2 and JNK are mostly responsible for the neural induction of ESCs [19, 36, 37]. Indeed, suppression of the ERK1/2 pathways enhanced the self-renewal of mouse ESCs [38] and retinoic acid-mediated neurogenesis required activation of ERK1/2 [37, 39]. In addition, activation of ERK1/2 by basic fibroblast growth factors contributed to neural growth and differentiation of mouse and human ESCs. In antioxidant enzyme $\operatorname{Prx} I^{--}$or $\operatorname{Prx} I^{-/}$cells, inhibition of JNK, but not ERK1/2, signaling provided the greatest protection against ROS-mediated loss of stemness, demonstrating that hyperactivation of JNK could override the action of ERK1/2 during neurogenesis under conditions of excessive ROS [19]. In this study, the role of Vps26a in neurogenesis mainly depended on ERK1/2 rather than JNK. Failure to activate the ERK1/2 cascade in differentiating $V p s 26 a^{-1-}$ ESCs resulted in prolonged maintenance of stemness and delayed neurogenesis (Fig. 3a, b), which was further evidenced by the mutual dependency between the Nox/ROS and ERK1/2 cascades during neurogenesis from ESCs. Thus, it was presumed that ERK1/2 functions as a central regulator of the stemness/differentiation transition via cooperation with the Nox/ROS cascade.

Nox also participates in the differentiation process of various cell types as a modulator of intracellular ROS signaling. Recent studies have shown that the redox status is closely associated with the commitment of stem cells to cardiac myocytes [40]. In addition, Nox4 activation is required for the differentiation of neonatal c-kit ${ }^{+}$cardiac precursor cells to smooth muscle cells [41]. Furthermore, BMP2 elevated ROS levels in neural crest stem cells (NCSCs), and treatment with a Nox inhibitor suppressed neuronal differentiation from NCSCs [22]. In particular, knockdown of Nox 4 appeared to be critical for the survival of NCSCs (at least in vitro), and Nox $4^{-1-}$ mouse embryos showed delayed overall development, indicating that Nox4-mediated ROS generation likely plays an important role in the differentiation of NCSCs during embryogenesis [22]. Our data are consistent with other reports, which describe retinoic acid (RA)-induced cell differentiation is characterized by the increase in Nox4 mRNA and protein expression [23, 42], and Nox4-generated ROS stimulatory effects on neural stem cell proliferation [43, 44]. Moreover, a role for ERK1/2 signaling pathway by retinoic acid that is required for neural differentiation has been already shown in ESCs [45, 46], and we confirmed that RA-induced upregulation of Nox 4 promotes the differentiation of ESCs into neural cells-activated MAPK kinase. The present study revealed that the defects of differentiating $V p s 26 a^{-1}$ ESCs may be closely related to reduced Nox4 levels. Experiments using a Nox inhibitor revealed that Vps26a-mediated neurogenesis from ESCs required the activation of Nox4. Interestingly, Nox4 and ERK1/2 activation were mutually dependent for successful neurogenesis, which is in good accordance with the results obtained from induction of neuronal differentiation by $\mathrm{H}_{2} \mathrm{O}_{2}$ concentration that was induced more in agreement with increased pERK1/2 (Supplementary Fig. 12). These results strongly suggest that Vps26a-mediated neurogenesis greatly depends on synergistic cooperation between Nox4, ROS, and ERK1/2.

We aimed to provide compelling evidence of a relationship between the retromer complex and ESC behavior, which led to the proposition of a comprehensive model and identification of the mechanism by which the stemness/differentiation transition is regulated via cooperation between Vps26a and redox signaling (Supplementary Fig. 13). In particular, activation of the Nox4/ROS/ERK1/2 pathway was severely impaired in differentiating Vps $26 a^{-/}$cells, suppressing ESCmediated neurogenesis. These findings represent new evidence of an association between Vps26a and redox signaling pathways, and may contribute to the development of alternative stem cell-based therapeutic strategies for the mass production of high-quality neurons.

\section{Materials and methods}

\section{Cell culture}

ESCs were grown in Dulbecco's modified Eagle's medium (DMEM; Invitrogen) containing 5\% ES-qualified fetal 
bovine serum (FBS; Invitrogen), 10\% FBS (Hyclone), 1× non-essential amino acids (NEAA; Invitrogen), $500 \mathrm{U} / \mathrm{ml}$ leukemia inhibitory factor (LIF; Chemicon, Temecula, CA, USA), and antibiotics (Invitrogen) on mitoticallyinactivated mouse embryonic fibroblasts (MIMEFs). The culture medium for P19 mouse embryonic carcinoma cells (ECCs) consisted of DMEM containing 10\% FBS (GIBCO) and antibiotics.

\section{Alkaline phosphatase activity assay}

All activity assay procedures were performed according to the manufacturer's instructions (Sigma-Aldrich, Leukocyte Alkaline Phosphatase Kit, Cat\# 86R). Briefly, after the culture medium had been discarded, the cells were fixed with citrate-acetone-formaldehyde fixative for $2 \mathrm{~min}$ at room temperature, and rinsed with distilled water. They were then incubated with $200 \mu \mathrm{g} / \mathrm{ml}$ Naphthol AS-MX phosphate (Sigma-Aldrich) and $1 \mathrm{mg} / \mathrm{ml}$ Fast Red TR salt (Sigma-Aldrich) in $100 \mathrm{mM}$ Tris buffer at $\mathrm{pH} 8.2$ for $15 \mathrm{~min}$ at room temperature. The staining reaction was terminated by rinsing with PBS.

\section{Embryoid body (EB) formation}

An ESC suspension $(10 \mathrm{ml})$ with a density of $10^{3}-10^{6}$ cells/ $\mathrm{ml}$ was seeded into a $100-\mathrm{mm}$ bacterial-grade dish. Seeded ESCs did not attach to the plastic surfaces of the bacterialgrade dishes, and naturally stuck to each other to form aggregates, without shaking [47]. The EB sizes were measured from collected micrographs using ImageJ software (developed at the U.S. National Institutes of Health and available at http://rsb.info.nih.gov/nih-image/).

\section{Semi-qPCR and real-time RT-qPCR}

Total RNAs were extracted from cells using the RNeasy plus mini kit (Qiagen) according to the manufacturer's instructions. Total RNA (1 $\mu \mathrm{g})$ was used for cDNA synthesis using ReverTra Ace- $\alpha$ (Toyobo). The expression levels of each gene were determined by semi-qPCR methods. The PCR was carried out in a $20 \mu$ reaction volume using the ExPrime Taq Master Mix (Genetbio). Each cycle consisted of a denaturation step at $94{ }^{\circ} \mathrm{C}$ for $20 \mathrm{~s}$, an annealing step at $62{ }^{\circ} \mathrm{C}$ for $20 \mathrm{~s}$, and an extension step at 72 ${ }^{\circ} \mathrm{C}$ for $20 \mathrm{~s}$. The final extension step was followed by a 5 min extension reaction at $72{ }^{\circ} \mathrm{C}$. Relative expression levels of the genes were measured by real-time RT-PCR using Brilliant III Ultra-Fast SYBR ${ }^{\circ}$ Green QPCR Master Mix (Stratagene) and analyzed with an Mx3000P QPCR systems (Stratagene). For the comparative analyses, mRNA expression levels were normalized to GAPDH and then expressed as the fold change. The sample delta $\mathrm{Ct}(\mathrm{S} \Delta \mathrm{Ct})$ value was calculated from the differences between the $\mathrm{Ct}$ values of GAPDH and the target genes. The relative gene expression levels between the samples and the controls were determined using the formula $2-(\mathrm{S} \Delta \mathrm{Ct}-\mathrm{C} \Delta \mathrm{Ct})$. PCR primers for amplification of the human and mouse cDNAs were designed in silico using Primer3 software (http://frodo. wi.mit.edu/primer3/input.htm; Supplementary information, Table S1).

\section{Western blot analysis}

For the western blot analysis, $30-60 \mu \mathrm{g}$ of protein lysates were separated in $8-12 \%$ sodium dodecyl sulfatepolyacrylamide gels and transferred onto nitrocellulose membranes (Millipore). The membranes were incubated overnight with primary antibodies (Supplementary Information, Table S2) at $4{ }^{\circ} \mathrm{C}$. The membranes were then washed five times with $10 \mathrm{mM}$ Tris- $\mathrm{HCl}$ (pH 7.5) containing $150 \mathrm{mM} \mathrm{NaCl}$ and $0.2 \%$ Tween-20 (TBST) and incubated with horseradish peroxidase-conjugated goat antirabbit IgG or anti-mouse $\operatorname{IgG}$ (1:5000, both from SigmaAldrich) for $1 \mathrm{~h}$ at room temperature. After the blots were washed with TBST, antibody binding was detected using a chemiluminescence detection system (Amersham) according to the manufacturer's instructions. Western blot band density was quantified with ImageJ software. Representative mean values were of at least three independent experiments with standard deviation.

\section{Double-label immunocytochemistry}

Cells were grown on a six-well cell culture slide, fixed with $4 \%$ formaldehyde (Sigma-Aldrich) at $4{ }^{\circ} \mathrm{C}$ for 30 min, permeabilized with $0.1 \%$ Triton $\mathrm{X}-100$ in phosphate-buffered saline (PBS), and blocked with $10 \%$ normal goat serum (Invitrogen) or 3\% bovine serum albumin (Sigma-Aldrich) for $1 \mathrm{~h}$ at room temperature. Subsequently, antibodies against Oct3/4 (SC-5279, Santa Cruz Biotechnology), Nanog (NBP2-19469, Novus Biological), MAP2 (AB5622, Millipore), Tubb3 (MAB1637, Millipore), pERK1/2 (4370, Cell Signaling), Hif2 $\alpha$ (NB100-122, Novus Biological), Nox4 (PA1-46014, Thermo Fisher Scientific), and Vps26a (AB23892, Abcam) were incubated with the prepared cells at $4{ }^{\circ} \mathrm{C}$ overnight. Finally, the cells were washed several times with $0.02 \%$ Tween-20 in PBS (PBST) and incubated with Alexa Fluor 488 or Alexa Fluor 594 secondary antibodies (Invitrogen). Fluorescence was analyzed by fluorescence microscopy (Leica). Quantification of the fluorescence intensity or positive cells/nuclear ratios was performed using ImageJ software. Representative mean values were of at least three independent experiments with standard deviation. 


\section{Plasmid construction}

The pcDNA6/Myc-His-Vps26a plasmid was constructed as follows. Full-length mouse Vps26a cDNA was amplified by PrimeSTAR ${ }^{\circ}$ HS DNA polymerase (TaKaRa) using mouse ESC cDNA as the template. The Vps26a-Myc-Hisexpressing plasmid was generated by ligating the resulting DNA into the pcDNA6/Myc-His vector (Invitrogen) following digestion with EcoRI and XhoI. The integrity of the Vps26a cDNA was confirmed by sequencing. The expression of Vps26a was analyzed by immunoblotting using specific Myc (TA150081, Origene) or Vps26a (AB23892, Abcam) antibodies. For construction of the C-terminally GST-tagged Vps26a, the cDNA encoding GST and Vps26a WT (1-360 aa), or deletion constructs containing amino acids $1-148,149-245$, or 246-360, were amplified and inserted into the pEBG-GST mammalian expression vector (Addgene \#22227). The HA-tagged Nox4C (C-terminal region, 249-574 aa) expression plasmids were kindly provided by Yun-Soo Bae (Ewha Woman's University, Seoul, Korea).

\section{Stable transfection of cells by lentiviral vector- mediated gene knockdown}

For the shRNA-mediated knockdown of Vps26a, pLKO.1 TRC library clones against Vps26a were used (SigmaAldrich, clone TRCN0000366609; 5'-AGG UGU UCA GCC AGA CCA C-3'), and a scramble non-silencing shRNA served as the control [pLKO.1-puro nonmammalian shRNA control (Sigma-Aldrich, SHC002V; 5'-CAA CAA GAT GAA GAG CAC CAA-3')]. P19 ECCs were stably transfected with the lentiviral vectors pLKO.1puro-CMV-Vps26a or pLKO.1-puro-shRNA Control. Stable cell lines were obtained after selection by long-term culture in medium containing $2 \mu \mathrm{g} / \mathrm{ml}$ puromycin (Invitrogen) for 20 days. Colonies were manually picked and further expanded in the presence of puromycin to establish individual knockdown lines. Each line was evaluated by RT-PCR and western blotting to confirm efficient knockdown of Vps26a.

\section{Chemical treatment}

To induce neurogenesis, ESCs were treated from day 0 to 6 with NBM (DMEM/F12 1:1 with N2 supplement and B27 supplement, GIBCO) and P19 ECCs using two differentiation methods: (1) cultured from day 0 to 6 with NBM and (2) treated from day 0 to 2 with NBM containing $0.5 \mu$ M RA (Sigma-Aldrich). For chemical treatment during neurogenesis, ESCs and P19 ECCs cells were treated with 50 and $100 \mu \mathrm{M}$ hydrogen peroxide $\left(\mathrm{H}_{2} \mathrm{O}_{2}\right.$; Sigma-Aldrich),
$2.5 \mathrm{mM}$ NAC (Sigma-Aldrich), and $10 \mu \mathrm{M}$ DPI (SigmaAldrich) for the indicated times, and MAPK inhibitors (all from Calbiochem), such as p38 MAPK (5 $\mu$ M SB203580), ERK1/2 (10 $\mu \mathrm{M}$ PD98059 or $10 \mu \mathrm{M}$ U0126), PI3K $(10 \mu \mathrm{M}$ LY294002), and JNK (5 $\mu$ M SP600125) were added at $12 \mathrm{~h}$ intervals. HEK293 cells were treated with $10 \mathrm{ng} / \mathrm{ml}$ epidermal growth factor (EGF, ERK 1/2 signaling activator; Sigma-Aldrich).

\section{Measurement of intracellular ROS}

Intracellular ROS generation was assessed using the ROS indicator 5,6-chloromethyl-2', $7^{\prime}$-dichlorodihydrofluorescein diacetate (CM- $\mathrm{H}_{2}$ DCFDA; Invitrogen). Exponentially growing cells were incubated with $5 \mu \mathrm{M} \mathrm{CM}-\mathrm{H}_{2}$ DCFDA at $37^{\circ} \mathrm{C}$ for $30 \mathrm{~min}$, washed twice with PBS, and centrifuged at $200 \times g$ for $5 \mathrm{~min}$. The DCFDA fluorescence intensity was measured immediately by flow cytometry with a FACSCalibur instrument (BD Biosciences) or by fluorescence microscopy (Zeiss Axiovert $200 \mathrm{M}$ ).

\section{Immunoprecipitation and GST pull-down assays}

Immunoprecipitations were performed as described previously [48]. Briefly, protein samples from $\sim 10^{7}$ cells were incubated with $3 \mu \mathrm{g}$ of specific antibody overnight at $4{ }^{\circ} \mathrm{C}$. The resulting immunocomplexes were then incubated with protein G-conjugated magnetic beads (DYNAL, Invitrogen) for $2 \mathrm{~h}$ at $4{ }^{\circ} \mathrm{C}$. The samples were washed four times with a digestion buffer supplemented with $0.1 \%$ NP-40 (protein lysis buffer) at room temperature. The proteins were then eluted by incubation with $0.4 \mathrm{M} \mathrm{NaCl}$ TE buffer for $30 \mathrm{~min}$ and analyzed by western blotting. GST pull-down assays were performed as previously described [49], with some modifications. Solubilized striatal extracts containing $50-100 \mu \mathrm{g}$ protein were diluted with $1 \times \mathrm{PBS} / 1 \%$ Triton $\mathrm{X}$ 100 , incubated with a $50 \%(\mathrm{v} / \mathrm{v})$ slurry of glutathioneSepharose 4B beads (GE Healthcare), and saturated with GST alone or GST-fusion protein $(5-10 \mu \mathrm{g})$ at $4{ }^{\circ} \mathrm{C}$ for $2 \mathrm{~h}$. The beads were then washed four times with $1 \times \mathrm{PBS} / 1 \%$ Triton X-100. All bound proteins were eluted with $4 \times$ lithium dodecyl sulfate loading buffer, resolved by SDSPAGE, and immunoblotted with specific antibodies.

\section{Teratoma assays}

Approximately $10^{7}$ cells were injected subcutaneously into the hind leg of $\mathrm{BALB} / \mathrm{c}$ nude mice. Teratomas were recovered when tumor formation became outwardly apparent. They were fixed in Bouin's fixative for hematoxylin and eosin staining or with paraformaldehyde for immunohistochemistry. 


\section{Immunohistochemistry}

Immunohistochemistry was performed as described previously [19]. Briefly, teratomas were fixed overnight with $10 \%$ neutral-buffered formalin, embedded in paraffin, and processed into 5-mm thick sections. For antigen retrieval, deparaffinized sections were heated for $4 \mathrm{~min}$ in a pressure cooker containing $10 \mathrm{mM}$ citrate buffer (pH 6.0). Subsequent procedures were performed at room temperature. Sections were pretreated with $3 \% \mathrm{H}_{2} \mathrm{O}_{2}$ in $0.1 \mathrm{M}$ TBS for $30 \mathrm{~min}$ to quench endogenous peroxidases and then treated with a protein blocking solution (Dako) for $20 \mathrm{~min}$. Next, they were incubated with antibodies against Oct3/4 (SC5279, Santa Cruz Biotechnology), Tubb3 (MAB1637, Millipore), Nox4 (14347-1-AP, Proteintech), and Vps26a (AB23892, Abcam) for $30 \mathrm{~min}$ in a humidified chamber. The sections were washed with TBST followed by incubation with EnVision anti-rabbit polymer (Dako) for $30 \mathrm{~min}$. The peroxidase bound to the antibody complex was visualized by treatment with 3,30-diaminobenzidine chromogen substrate solution (Dako). The color reaction was monitored under a microscope to determine the optimal incubation time, and the reaction was stopped by several washes with TBS. Immunolabeled sections were dehydrated in a graded ethanol series, defatted in xylene, mounted, and examined under a bright-field using an Olympus BX51 microscope (Olympus). Images were acquired using an Olympus DP 70 camera.

\section{Statistical analyses}

The data are the means and standard deviation (SD) from three independent experiments. Differences among groups were calculated for significance $(P<0.05)$ using Prism software. Experimental differences between two groups were calculated via the two-tailed $t$ test, and differences among three or more groups were calculated via one-way analysis of variance and the Bonferroni post-hoc test. $P<$ 0.05 was considered to indicate statistical significance and is indicated on graphs by an asterisk. $P<0.01$ and $<0.001$ are indicated by two and three asterisks, respectively.

Acknowledgements This study was supported by grants from the KRIBB Research Initiative Program (KGM4251824) and the Bio \& Medical Technology Development Program through the National Research Foundation of Korea (NRF) funded by the Ministry of Education, Science and Technology (MEST) (No. 2018M3A9H1023142), Republic of Korea.

Author contributions S-AC, Y-HK, and YHP designed and performed most of the experiments, analyzed data and wrote the paper. B-SS, HJY, P-SJ, and S-BY performed all PCR and western blot analysis. JHL and Y-SB helped find the preliminary phenomenon. I-SS, J-JC, J$\mathrm{SK}$, and J-MK participated in screening for tissue analysis. J-WH and M-KK performed ES cells-related experiments. B-WS designed vector construction. S-UK and K-TC supervised the study, and S-RL, KI wrote the paper together with S-UK and K-TC.

\section{Compliance with ethical standards}

Conflict of interest The authors declare that they have no conflict of interest.

Open Access This article is licensed under a Creative Commons Attribution 4.0 International License, which permits use, sharing, adaptation, distribution and reproduction in any medium or format, as long as you give appropriate credit to the original author(s) and the source, provide a link to the Creative Commons license, and indicate if changes were made. The images or other third party material in this article are included in the article's Creative Commons license, unless indicated otherwise in a credit line to the material. If material is not included in the article's Creative Commons license and your intended use is not permitted by statutory regulation or exceeds the permitted use, you will need to obtain permission directly from the copyright holder. To view a copy of this license, visit http://creativecommons. org/licenses/by/4.0/.

\section{References}

1. Evans MJ, Kaufman MH. Establishment in culture of pluripotential cells from mouse embryos. Nature. 1981;292:154-6.

2. Gallon M, Cullen PJ. Retromer and sorting nexins in endosomal sorting. Biochem Soc Trans. 2015;43:33-47.

3. Seaman MN. The retromer complex - endosomal protein recycling and beyond. J Cell Sci. 2012;125(Pt 20):4693-702.

4. Chamberland JP, Ritter B. Retromer revisited: evolving roles for retromer in endosomal sorting. J Cell Biol. 2017;216:3433-6.

5. Wang S, Bellen HJ. The retromer complex in development and disease. Development. 2015;142:2392-6.

6. Zimprich A, Benet-Pages A, Struhal W, Graf E, Eck SH, Offman $\mathrm{MN}$, et al. A mutation in VPS35, encoding a subunit of the retromer complex, causes late-onset Parkinson disease. Am J Hum Genet. 2011;89:168-75.

7. Choy RW, Park M, Temkin P, Herring BE, Marley A, Nicoll RA, et al. Retromer mediates a discrete route of local membrane delivery to dendrites. Neuron. 2014;82:55-62.

8. Vardarajan BN, Bruesegem SY, Harbour ME, Inzelberg R, Friedland R, St George-Hyslop P, et al. Identification of Alzheimer disease-associated variants in genes that regulate retromer function. Neurobiol Aging. 2012;33:2231 e2215-2231 e2230.

9. Griffin CT, Trejo J, Magnuson T. Genetic evidence for a mammalian retromer complex containing sorting nexins 1 and 2 . Proc Natl Acad Sci USA. 2005;102:15173-7.

10. Radice G, Lee JJ, Costantini F. H beta 58, an insertional mutation affecting early postimplantation development of the mouse embryo. Development. 1991;111:801-11.

11. Lee JJ, Radice G, Perkins CP, Costantini F. Identification and characterization of a novel, evolutionarily conserved gene disrupted by the murine $\mathrm{H}$ beta 58 embryonic lethal transgene insertion. Development. 1992;115:277-88.

12. Muhammad A, Flores I, Zhang $\mathrm{H}, \mathrm{Yu} \mathrm{R}$, Staniszewski A, Planel E, et al. Retromer deficiency observed in Alzheimer's disease causes hippocampal dysfunction, neurodegeneration, and Abeta accumulation. Proc Natl Acad Sci USA. 2008;105: 7327-32.

13. Espada J. Current methods to unravel ROS biology. Methods. 2016;109:1-2.

14. Lewandowski D, Barroca V, Duconge F, Bayer J, Van Nhieu JT, Pestourie $\mathrm{C}$, et al. In vivo cellular imaging pinpoints the role of 
reactive oxygen species in the early steps of adult hematopoietic reconstitution. Blood. 2010;115:443-52.

15. Nayernia Z, Jaquet V, Krause KH. New insights on NOX enzymes in the central nervous system. Antioxid Redox Signal. 2014;20:2815-37.

16. Schmelter M, Ateghang B, Helmig S, Wartenberg M, Sauer H. Embryonic stem cells utilize reactive oxygen species as transducers of mechanical strain-induced cardiovascular differentiation. FASEB J. 2006;20:1182-4.

17. Ji AR, Ku SY, Cho MS, Kim YY, Kim YJ, Oh SK, et al. Reactive oxygen species enhance differentiation of human embryonic stem cells into mesendodermal lineage. Exp Mol Med. 2010;42:17586.

18. Binetruy B, Heasley L, Bost F, Caron L, Aouadi M. Concise review: regulation of embryonic stem cell lineage commitment by mitogen-activated protein kinases. Stem Cells. 2007;25: 1090-5.

19. Kim SU, Park YH, Kim JM, Sun HN, Song IS, Huang SM, et al. Dominant role of peroxiredoxin/JNK axis in stemness regulation during neurogenesis from embryonic stem cells. Stem Cells. 2014;32:998-1011.

20. Fuse A, Furuya N, Kakuta S, Inose A, Sato M, Koike M, et al. VPS29-VPS35 intermediate of retromer is stable and may be involved in the retromer complex assembly process. FEBS Lett. 2015;589:1430-6.

21. Morabito MV, Berman DE, Schneider RT, Zhang Y, Leibel RL, Small SA. Hyperleucinemia causes hippocampal retromer deficiency linking diabetes to Alzheimer's disease. Neurobiol Dis. 2014;65:188-92.

22. Lee JE, Cho KE, Lee KE, Kim J, Bae YS. Nox4-mediated cell signaling regulates differentiation and survival of neural crest stem cells. Mol Cells. 2014;37:907-11.

23. Borquez DA, Urrutia PJ, Wilson C, van Zundert B, Nunez MT, Gonzalez-Billault C. Dissecting the role of redox signaling in neuronal development. J Neurochem. 2016;137:506-17.

24. Small SA, Petsko GA. Retromer in Alzheimer disease, Parkinson disease and other neurological disorders. Nat Rev Neurosci. 2015;16:126-32.

25. Li C, Shah SZ, Zhao D, Yang L. Role of the retromer complex in neurodegenerative diseases. Front Aging Neurosci. 2016;8:42.

26. Kinoshita A, Fukumoto H, Shah T, Whelan CM, Irizarry MC, Hyman BT. Demonstration by FRET of BACE interaction with the amyloid precursor protein at the cell surface and in early endosomes. J Cell Sci. 2003;116(Pt 16):3339-46.

27. Small SA, Kent K, Pierce A, Leung C, Kang MS, Okada H, et al. Model-guided microarray implicates the retromer complex in Alzheimer's disease. Ann Neurol. 2005;58:909-19.

28. He X, Li F, Chang WP, Tang J. GGA proteins mediate the recycling pathway of memapsin 2 (BACE). J Biol Chem. 2005;280:11696-703.

29. Young JE, Fong LK, Frankowski H, Petsko GA, Small SA, Goldstein LSB. Stabilizing the retromer complex in a human stem cell model of Alzheimer's disease reduces TAU phosphorylation independently of amyloid precursor protein. Stem Cell Rep. 2018;10:1046-58.

30. Vazquez-Sanchez S, Bobeldijk S, Dekker MP, van Keimpema L, van Weering JRT. VPS35 depletion does not impair presynaptic structure and function. Sci Rep. 2018;8:2996.

31. Zhou L, Wang W, Hoppel C, Liu J, Zhu X. Parkinson's diseaseassociated pathogenic VPS35 mutation causes complex I deficits. Biochim Biophys Acta. 2017;1863:2791-5.

32. Zavodszky E, Seaman MN, Moreau K, Jimenez-Sanchez M, Breusegem SY, Harbour ME, et al. Mutation in VPS35 associated with Parkinson's disease impairs WASH complex association and inhibits autophagy. Nat Commun. 2014;5:3828.

33. Cassarino DS, Fall CP, Swerdlow RH, Smith TS, Halvorsen EM, Miller SW, et al. Elevated reactive oxygen species and antioxidant enzyme activities in animal and cellular models of Parkinson's disease. Biochim Biophys Acta. 1997;1362:77-86.

34. Esposito E, Cuzzocrea S. New therapeutic strategy for Parkinson's and Alzheimer's disease. Curr Med Chem. 2010;17:2764-74.

35. Sun HN, Kim SU, Lee MS, Kim SK, Kim JM, Yim M, et al. Nicotinamide adenine dinucleotide phosphate (NADPH) oxidasedependent activation of phosphoinositide 3-kinase and p38 mitogen-activated protein kinase signal pathways is required for lipopolysaccharide-induced microglial phagocytosis. Biol Pharm Bull. 2008;31:1711-5.

36. Hackett JA, Surani MA. Regulatory principles of pluripotency: from the ground state up. Cell Stem Cell. 2014;15:416-30.

37. Li Z, Theus MH, Wei L. Role of ERK 1/2 signaling in neuronal differentiation of cultured embryonic stem cells. Dev Growth Differ. 2006;48:513-23.

38. Kim MO, Kim SH, Cho YY, Nadas J, Jeong CH, Yao K, et al. ERK1 and ERK2 regulate embryonic stem cell self-renewal through phosphorylation of Klf4. Nat Struct Mol Biol. 2012;19:283-90.

39. Sartore RC, Campos PB, Trujillo CA, Ramalho BL, Negraes PD, Paulsen BS, et al. Retinoic acid-treated pluripotent stem cells undergoing neurogenesis present increased aneuploidy and micronuclei formation. PLoS ONE. 2011;6:e20667.

40. Buggisch M, Ateghang B, Ruhe C, Strobel C, Lange S, Wartenberg $\mathrm{M}$, et al. Stimulation of ES-cell-derived cardiomyogenesis and neonatal cardiac cell proliferation by reactive oxygen species and NADPH oxidase. J Cell Sci. 2007;120(Pt 5):885-94.

41. Nadworny AS, Guruju MR, Poor D, Doran RM, Sharma RV, Kotlikoff MI, et al. Nox2 and Nox4 influence neonatal c-kit( $(+)$ cardiac precursor cell status and differentiation. Am J Physiol Heart Circ Physiol. 2013;305:H829-842.

42. Nitti M, Furfaro AL, Cevasco C, Traverso N, Marinari UM, Pronzato MA, et al. PKC delta and NADPH oxidase in retinoic acid-induced neuroblastoma cell differentiation. Cell Signal. 2010;22:828-35.

43. Bigarella CL, Liang R, Ghaffari S. Stem cells and the impact of ROS signaling. Development. 2014;141:4206-18.

44. Topchiy E, Panzhinskiy E, Griffin WS, Barger SW, Das M, Zawada WM. Nox4-generated superoxide drives angiotensin IIinduced neural stem cell proliferation. Dev Neurosci. 2013;35:293-305.

45. Hamilton WB, Brickman JM. Erk signaling suppresses embryonic stem cell self-renewal to specify endoderm. Cell Rep. 2014;9:2056-70.

46. Hou PS, Huang WC, Chiang W, Lin WC, Chien CL. Impaired neural differentiation potency by retinoic acid receptor-alpha pathway defect in induced pluripotent stem cells. Cell Reprogram. 2014:16:467-76.

47. Kurosawa H. Methods for inducing embryoid body formation: in vitro differentiation system of embryonic stem cells. J Biosci Bioeng. 2007;103:389-98.

48. Neri F, Krepelova A, Incarnato D, Maldotti M, Parlato C, Galvagni $\mathrm{F}$, et al. Dnmt3L antagonizes DNA methylation at bivalent promoters and favors DNA methylation at gene bodies in ESCs. Cell. 2013;155:121-34.

49. Jin DZ, Guo ML, Xue B, Fibuch EE, Choe ES, Mao LM, et al. Phosphorylation and feedback regulation of metabotropic glutamate receptor 1 by calcium/calmodulin-dependent protein kinase II. J Neurosci. 2013;33:3402-12. 


\section{Affiliations}

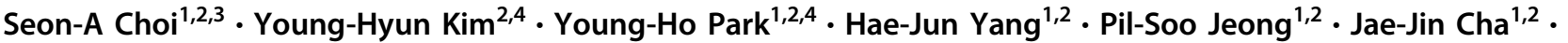

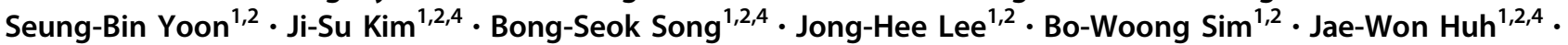 In-Sung Song ${ }^{5}$. Sang-Rae Lee ${ }^{1,2,4} \cdot$ Min-Kyu Kim $^{3} \cdot$ Jin-Man Kim ${ }^{6}$ - Yun Soo Bae ${ }^{7}$ Kazuhiko Imakawa ${ }^{8,9}$. Sun-Uk Kim ${ }^{1,2,4} \cdot$ Kyu-Tae Chang ${ }^{2,4}$}

1 Futuristic Animal Resource \& Research Center, Korea Research Institute of Bioscience and Biotechnology, Chungcheongbukdo 28116, Republic of Korea

2 National Primate Research Center, Korea Research Institute of Bioscience and Biotechnology, Chungcheongbuk-do 28116, Republic of Korea

3 Laboratory of Animal Reproduction and Physiology, Department of Animal Science and Biotechnology, College of Agriculture and Life Science, Chungnam National University, Daejeon 34134, Republic of Korea

4 Department of Functional Genomics, University of Science and Technology, Daejeon 34113, Republic of Korea
5 Department of Biomedical Sciences, College of Medicine, Ulsan University, Asan Medical Center, Seoul 05505, Republic of Korea

6 College of Medicine, Chungnam National University, Daejeon 34134, Republic of Korea

7 Department of Life Science, Ewha Womans University, Seoul 03760, Republic of Korea

8 Animal Resource Science Center, Graduate School of Agricultural and Life Sciences, The University of Tokyo, Ibaraki 319-0206, Japan

9 Institute of Agricultural Sciences, Tokai University, Kumamoto 862-8652, Japan 